Article

\title{
Optimal Bail-Out Dividend Problem with Transaction Cost and Capital Injection Constraint
}

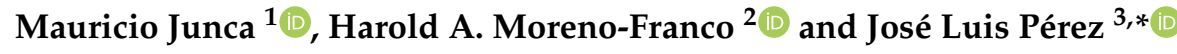 \\ 1 Department of Mathematics, Universidad de los Andes, Bogotá 11711, Colombia; \\ mj.junca20@uniandes.edu.co \\ 2 Department of Mathematics and Statistics, Universidad del Norte, Barranquilla 080003, Colombia; \\ hamoreno@uninorte.edu.co \\ 3 Department of Probability and Statistics, Centro de Investigación en Matemáticas A.C., \\ Guanajuato 36000, Mexico \\ * Correspondence: jluis.garmendia@cimat.mx
}

Received: 18 December 2018; Accepted: 29 January 2019; Published: 31 January 2019

\begin{abstract}
We consider the optimal bail-out dividend problem with fixed transaction cost for a Lévy risk model with a constraint on the expected present value of injected capital. To solve this problem, we first consider the optimal bail-out dividend problem with transaction cost and capital injection and show the optimality of reflected $\left(c_{1}, c_{2}\right)$-policies. We then find the optimal Lagrange multiplier, by showing that in the dual Lagrangian problem the complementary slackness conditions are met. Finally, we present some numerical examples to support our results.
\end{abstract}

Keywords: dividend payment; optimal control; capital injection constraint; spectrally negative Lévy processes; reflected Lévy processes; scale functions

MSC: 60G51; 91B30

\section{Introduction}

De Finetti introduced in 1957 the expected net present value (NPV) of dividends paid by an insurance company as a criterion for assessing its stability. According to this model, the maximum of the expected NPVs, if it exists, can be a proxy for the insurance company's value. In some cases (e.g., due to regulatory issues), the insurance company has to ensure the negative balance protection and therefore must be rescued by injecting capital. Hence, the company aims to maximize the total amount of expected dividend payments minus the total expected cost of capital injection while permanently keeping the surplus process non-negative.

Usually, spectrally negative Lévy processes (Lévy processes with only downward jumps) are used to model the underlying surplus process of an insurance company, which increases with premium payments and decreases with insurance payouts. The optimization problem for this model was studied by Avram et al. (2007), who proved that it is optimal to inject capital when the process is below zero and pay dividends when the process is above a suitably chosen threshold.

In this paper, we focus on the case when the insurance company pays a fixed transaction cost each time a dividend payment is made. The fixed transaction cost makes the continuous payment of dividends no longer feasible, which implies that only lump sum dividend payments are possible. In this case, a strategy is assumed to have the form of impulse control; whenever dividends are accrued, a constant transaction cost $\delta>0$ is incurred. Unlike the barrier strategies described above, which are typically optimal for the case without transaction cost, we pursue the optimality of the reflected $\left(c_{1}, c_{2}\right)$-policies. In these strategies, the surplus process is brought down to $c_{1}$ whenever it 
exceeds the level $c_{2}$ for some $0 \leq c_{1}<c_{2}<\infty$, and pushes the surplus to 0 whenever it goes below 0 . Previously, the version of the de Finetti's optimal dividend problem with fixed transaction cost and without bail-outs was solved for the spectrally negative case by Loeffen (2009) and for the dual model (i.e., spectrally positive Lévy processes) by Bayraktar et al. (2014b).

In this paper, we also propose a model to maximize the value of the insurance company by means of the dividend payments while keeping the expected present value of the capital injection bounded. The idea of introducing this constraint is to bound the budget needed for the company to survive and therefore to reduce the risk faced (e.g., operational risk).

Specifically, we solve the following two problems:

1. We find the solution to the optimal bail-out dividend problem with fixed transaction cost for the case of spectrally negative Lévy processes. We show that a reflected $\left(c_{1}, c_{2}\right)$-policy is optimal (see Theorem 1). We use scale functions to characterize the optimal thresholds as well as the value function. We prove the optimality of the proposed policy by means of a verification theorem.

2. We solve the constrained dividend maximization problem with capital injection on the set of strategies such that the expected net present value of injected capital must be bounded by a given constant. This is an offshoot of Hernández et al. (2018) for the bail-out case. Using the previous results, in Theorems 2 and 3, we present the solution when the surplus of the company is modeled by a spectrally negative Lévy process.

This paper is organized as follows. In Section 2, we introduce the problem. In Section 3, we provide a review of scale functions and some fluctuation identities of spectrally negative Lévy processes as well as their reflected versions. In Section 4, we solve the optimal bail-out dividend problem with fixed transaction cost for the case of a spectrally negative Lévy process. In Section 5, we present the solution for the constrained bail-out dividend problem. In Section 6, we illustrate our main results by giving some numerical examples.

\section{Formulation of the Problem}

Let $X=\left\{X_{t}: t \geq 0\right\}$ be a Lévy process defined on a probability space $(\Omega, \mathcal{F}, \mathbb{P})$, and let $\mathbb{F}:=\left\{\mathcal{F}_{t}: t \geq 0\right\}$ be the completed and right-continuous filtration generated by $X$. Recall that a Lévy process is a process that has càdlàg paths and stationary and independent increments. For $x \in \mathbb{R}$, we denote by $\mathbb{P}_{x}$ the law of $X$, where $X_{0}=x$. For convenience, we take $\mathbb{P}_{0} \equiv \mathbb{P}$, when $x=0$. The expectation operator associated with $\mathbb{P}_{x}$ is denoted by $\mathbb{E}_{x}$. We take $\mathbb{E}_{0} \equiv \mathbb{E}$, where $\mathbb{E}$ is the expectation operator associated with $\mathbb{P}$.

We henceforth assume that the insurance company's surplus $X$ is modeled by a spectrally negative process, i.e., a Lévy process that only has negative jumps. We omit the case when $X$ has monotone trajectories to avoid trivial cases.

The Laplace exponent of $X$ is given by

$$
\psi(\theta):=\log \mathbb{E}\left[\theta X_{1}\right]=\gamma \theta+\frac{\sigma^{2}}{2} \theta^{2}-\int_{(0, \infty)}\left(1-\mathrm{e}^{-\theta z}-\theta z \mathbf{1}_{\{0<z \leq 1\}}\right) \Pi(\mathrm{d} z), \quad \theta \geq 0,
$$

where $\gamma \in \mathbb{R}, \sigma \geq 0$, and the Lévy measure of $X, \Pi$, is a measure defined on $(0, \infty)$ satisfying

$$
\int_{(0, \infty)}\left(1 \wedge z^{2}\right) \Pi(\mathrm{d} z)<\infty
$$

As is well-known, the process $X$ has bounded variation paths if and only if $\sigma=0$ and $\int_{(0,1]} z \Pi(\mathrm{d} z)<\infty$. In this case, $X$ can be written as

$$
X_{t}=c t-\widetilde{S}_{t}, \quad t \geq 0,
$$


where $c:=\gamma+\int_{(0,1]} z \Pi(\mathrm{d} z)$ and $\widetilde{S}=\left\{\widetilde{S}_{t}: t \geq 0\right\}$ is a drift-less subordinator. Since we omit the case when $X$ has monotone paths, it is necessary that the constant $c$ is greater than zero. Note that the Laplace exponent of $X$, with $X$ as in Equation (1), is given as follows,

$$
\psi(\theta)=c \theta-\int_{(0, \infty)}\left(1-\mathrm{e}^{-\theta z}\right) \Pi(\mathrm{d} z), \quad \theta \geq 0 .
$$

De Finetti's Problem with Fixed Transaction Cost and Capital Injection

Let $\pi=\left\{L^{\pi}, R^{\pi}\right\}$ be a strategy, where $L^{\pi}$ is left-continuous $\mathbb{P}_{x}$-a.s., and $R^{\pi}$ is right-continuous $\mathbb{P}_{x}$-a.s. Additionally, we assume that $L^{\pi}$ and $R^{\pi}$ are non-negative, and non-decreasing $\mathbb{P}_{x}$-a.s., start at zero and are adapted to the filtration $\mathbb{F}$. Then, the controlled process, $X^{\pi}$, associated with the strategy $\pi$, is the following

$$
X_{t}^{\pi}=X_{t}-L_{t}^{\pi}+R_{t}^{\pi}, \quad t \geq 0 .
$$

For each $t \geq 0$, the quantities $L_{t}^{\pi}$ and $R_{t}^{\pi}$ represent the cumulative amounts that the insurance company has paid to its shareholders and has injected, respectively.

The set of admissible policies $\Theta$ consists of those policies $\pi$ for which $X^{\pi}$ is non-negative and for $x \geq 0$,

$$
\mathbb{E}_{x}\left[\int_{0}^{\infty} \mathrm{e}^{-q t} \mathrm{~d} R_{t}^{\pi}\right]<\infty
$$

When there is a fixed transaction $\operatorname{cost} \delta>0$, we only consider the class of admissible strategies $\pi=\left\{L^{\pi}, R^{\pi}\right\} \in \Theta$ such that

$$
L_{t}^{\pi}=\sum_{0 \leq s \leq t} \Delta L_{s}^{\pi}, \quad t \geq 0,
$$

where $\Delta L_{t}^{\pi}:=L_{t+}^{\pi}-L_{t}^{\pi}$. We denote this class by $\Theta_{\delta}$ and in the case $\delta=0$, we take $\Theta_{0} \equiv \Theta$.

Given an initial capital $x \geq 0$ and a policy $\pi=\left\{L^{\pi}, R^{\pi}\right\} \in \Theta_{\delta}$, with $\delta \geq 0$, we define the expected NPV as follows,

$$
v_{\delta, \Lambda}^{\pi}(x):=\mathbb{E}_{x}\left[\int_{0}^{\infty} \mathrm{e}^{-q t} \mathrm{~d}\left(L_{t}^{\pi}-\delta \sum_{0 \leq s \leq t} \mathbf{1}_{\left\{\Delta L_{s}^{\pi}>0\right\}}\right)-\Lambda \int_{0}^{\infty} \mathrm{e}^{-q t} \mathrm{~d} R_{t}^{\pi}\right],
$$

where $q>0, \delta \geq 0$, and $\Lambda>0$ is the unit cost per capital injected.

Remark 1. Note that in the case of proportional transaction cost the expected NPV changes to

$$
\mathbb{E}_{x}\left[\int_{0}^{\infty} \mathrm{e}^{-q t} \mathrm{~d}\left(\beta L_{t}^{\pi}-\delta \sum_{0 \leq s \leq t} \mathbf{1}_{\left\{\Delta L_{s}^{\pi}>0\right\}}\right)-\Lambda \int_{0}^{\infty} \mathrm{e}^{-q t} \mathrm{~d} R_{t}^{\pi}\right],
$$

where $0<\beta<1$, so by changing $\delta$ and $\Lambda$ appropriately we can recover Equation (2).

Hence, the value function we aim to find is

$$
V_{\delta, \Lambda}(x):=\sup _{\pi \in \Theta_{\delta}} v_{\delta, \Lambda}^{\pi}(x) .
$$

Remark 2. Since we want to avoid this function taking the value $-\infty$, we assume that $\psi^{\prime}(0+)=\mathbb{E}\left[X_{1}\right]>-\infty$. We also assume that $\Lambda \geq 1$, otherwise the value function will go to infinity since large amounts of dividends will be paid, given that the company will inject capital at a cheaper cost to bail out.

Note that the problem in Equation (3) was studied by Avram et al. (2007) under the assumption $\delta=0$ (see Section 3.2). Therefore, we focus on the optimal control problem when $\delta>0$ (see Section 4 ). 


\section{Preliminaries}

In this section, we revise the scale functions of spectrally negative Lévy processes and their properties (see, e.g., Kuznetsov et al. (2013); Kyprianou (2014)). We also recall well known results regarding optimal dividend strategies with capital injection for spectrally one-sided Lévy processes when the transaction cost is equal to 0 (i.e., $\delta=0$ ).

For each $q \geq 0$, there exists a map $W^{(q)}: \mathbb{R} \longrightarrow[0, \infty)$, called $q$-scale function, satisfying $W^{(q)}(x)=$ 0 for $x \in(-\infty, 0)$, and strictly increasing on $[0, \infty)$, which is defined by its Laplace transform:

$$
\int_{0}^{\infty} \mathrm{e}^{-\theta x} W^{(q)}(x) \mathrm{d} x=\frac{1}{\psi(\theta)-q}, \quad \theta>\Phi(q),
$$

where

$$
\Phi(q):=\sup \{\lambda \geq 0: \psi(\lambda)=q\}
$$

We also define, for $x \in \mathbb{R}$,

$$
\begin{aligned}
& \bar{W}^{(q)}(x):=\int_{0}^{x} W^{(q)}(y) \mathrm{d} y, \quad Z^{(q)}(x):=1+q \bar{W}^{(q)}(x), \\
& \bar{Z}^{(q)}(x):=\int_{0}^{x} Z^{(q)}(z) \mathrm{d} z=x+q \int_{0}^{x} \int_{0}^{z} W^{(q)}(w) \mathrm{d} w \mathrm{~d} z .
\end{aligned}
$$

Since $W^{(q)}$ is equal to zero on $(-\infty, 0)$, we have

$$
\bar{W}^{(q)}(x)=0, \quad Z^{(q)}(x)=1 \quad \text { and } \quad \bar{Z}^{(q)}(x)=x, \quad x \leq 0 .
$$

\section{Remark 3.}

1. By Equation (8.26) of Kyprianou (2014), the left-and right-hand derivatives of $W^{(q)}$ always exist on $\mathbb{R} \backslash\{0\}$. In addition, as in, e.g., (Chan et al. 2011, Theorem 3), if $X$ is of unbounded variation or the Lévy measure is atomless, we have $W^{(q)} \in C^{1}(\mathbb{R} \backslash\{0\})$.

2. From Lemmas 3.1-3.2 of Kuznetsov et al. (2013), we know

$$
\begin{gathered}
W^{(q)}(0)= \begin{cases}0, & \text { if } X \text { is of unbounded variation, } \\
\frac{1}{c^{\prime}}, & \text { if } X \text { is of bounded variation, }\end{cases} \\
W^{(q) \prime}(0+):=\lim _{x \downarrow 0} W^{(q) \prime}(x)= \begin{cases}\frac{2}{\sigma^{2}}, & \text { if } \sigma>0, \\
\infty, & \text { if } \sigma=0 \text { and } \Pi(0, \infty)=\infty, \\
\frac{q+\Pi(0, \infty)}{c^{2}}, & \text { if } \sigma=0 \text { and } \Pi(0, \infty)<\infty .\end{cases}
\end{gathered}
$$

3. From Lemma 3.3 of Kuznetsov et al. (2013), $W_{\Phi(q)}(x):=\mathrm{e}^{-\Phi(q) x} W^{(q)}(x) \nearrow \psi^{\prime}(\Phi(q))^{-1}$, as $x \uparrow \infty$.

Due to Remark 3, we make the following assumption throughout the paper.

Assumption 1. We assume that either $X$ has unbounded variation or $\Pi$ is absolutely continuous with respect to the Lebesgue measure. Under this assumption, it holds that $W^{(q)}$ is $C^{1}$ in $(0, \infty)$.

We give the following properties related to $Z^{(q)}$ and $\bar{W}^{(q)}$ for later use.

\section{Remark 4.}

(i) By Proposition 5.5 in Hernández et al. (2018), we have that $Z^{(q)}$ is a strictly log-convex function on $(0, \infty)$, for $q>0$.

(ii) From Lemma 1 in Avram et al. (2007), it is known that $\bar{W}^{(q)}$ is a log-concave function on $(0, \infty)$. 
We define the stopping times $\tau_{a^{-}}$and $\tau_{a^{+}}$, respectively, as follows,

$$
\tau_{a}^{-}:=\inf \left\{t>0: X_{t}<a\right\} \quad \text { and } \quad \tau_{a}^{+}:=\inf \left\{t>0: X_{t}>a\right\}, \quad a \in \mathbb{R} ;
$$

here and further on, let inf $\varnothing=\infty$. By Theorem 8.1 in Kyprianou (2014), we have that

$$
\begin{aligned}
& \mathbb{E}_{x}\left[\mathrm{e}^{-q \tau_{a}^{+}} \mathbf{1}_{\left\{\tau_{a}^{+}<\tau_{b}^{-}\right\}}\right]=\frac{W^{(q)}(x-b)}{W^{(q)}(a-b)}, \\
& \mathbb{E}_{x}\left[\mathrm{e}^{-q \tau_{b}^{-}} \mathbf{1}_{\left\{\tau_{a}^{+}>\tau_{b}^{-}\right\}}\right]=Z^{(q)}(x-b)-Z^{(q)}(a-b) \frac{W^{(q)}(x-b)}{W^{(q)}(a-b)},
\end{aligned}
$$

\subsection{Reflected Lévy Processes}

Let $S=\left\{S_{t}: t \geq 0\right\}$ and $R^{0}=\left\{R_{t}^{0}: t \geq 0\right\}$ be defined, respectively, as

$$
S_{t}:=\sup _{0 \leq s \leq t}\left(X_{s} \vee 0\right) \quad \text { and } \quad R_{t}^{0}:=\sup _{0 \leq s \leq t}\left(-X_{s} \vee 0\right) .
$$

We denote $\hat{Y}:=S-X$ and $Y:=X+R^{0}$, which are strong Markov processes. Observe that the process $R^{0}$ pushes $X$ upwards whenever it attempts to down-cross the level 0; as a result the process $Y$ only takes values on $[0, \infty)$. An introduction to the theory of Lévy processes and their reflected processes can be encountered in Bertoin (1998); Kyprianou (2014).

Let $\hat{\tau}_{a}$ be defined as $\hat{\tau}_{a}=\inf \left\{t>0: \hat{Y}_{t} \in(a, \infty)\right\}$, with $a>0$. Then, by Proposition 2 in Pistorius (2004),

$$
\mathbb{E}_{-x}\left[\mathrm{e}^{-q \hat{\tau}_{a}}\right]=Z^{(q)}(a-x)-q W^{(q)}(a-x) \frac{W^{(q)}(a)}{W^{(q) \prime}(a)}, \quad x \in[0, a] .
$$

We define for $a>0$,

$$
H(a):=\mathbb{E}_{0}\left[\mathrm{e}^{-q \hat{t}_{a}}\right]=Z^{(q)}(a)-q \frac{\left[W^{(q)}(a)\right]^{2}}{W^{(q) \prime}(a)} .
$$

Remark 5. Note that, by definition, the function $H$ is strictly positive, strictly decreasing and satisfies

$$
\lim _{a \rightarrow \infty} H(a)=0, \quad \lim _{a \rightarrow 0} H(a)=1-\frac{q\left[W^{(q)}(0)\right]^{2}}{W^{(q) \prime}(0+)} .
$$

Therefore, the function $H$ has an inverse from $(0,1-q /(q+\Pi(0, \infty)))$ onto $(0, \infty)$ when $\sigma=0$ and $\Pi(0, \infty)<\infty$, and from $(0,1)$ onto $(0, \infty)$ otherwise.

Similarly, taking $\kappa_{b}:=\inf \left\{t>0: Y_{t} \in(b, \infty)\right\}$, with $b>0$, we know from Proposition 2 in Pistorius (2004) that

$$
\mathbb{E}_{x}\left[\mathrm{e}^{-q \kappa_{b}}\right]=\frac{Z^{(q)}(x)}{Z^{(q)}(b)}, \quad x \leq b
$$


In addition, we know from (Avram et al. 2007, page 167) that

$$
\begin{aligned}
\mathbb{E}_{x}\left[\int_{\left[0, \kappa_{b}\right]} \mathrm{e}^{-q t} \mathrm{~d} R_{t}^{0}\right]= & -\bar{Z}^{(q)}(x)+\Phi(q)^{-1} Z^{(q)}(x)-\frac{\psi^{\prime}(0+)}{q} \\
& +\left(\bar{Z}^{(q)}(b)-\Phi(q)^{-1} Z^{(q)}(b)+\frac{\psi^{\prime}(0+)}{q}\right) \frac{Z^{(q)}(x)}{Z^{(q)}(b)} \\
= & -k^{(q)}(x)+\frac{Z^{(q)}(x)}{Z^{(q)}(b)} k^{(q)}(b), \quad x \leq b,
\end{aligned}
$$

where

$$
k^{(q)}(x):=\bar{Z}^{(q)}(x)+\frac{\psi^{\prime}(0+)}{q} .
$$

\subsection{Optimal Dividends without Transaction Cost and with Capital Injection}

When $\delta=0$, Equation (2) becomes

$$
v_{\Lambda}^{\pi}(x):=v_{0, \Lambda}^{\pi}(x)=\mathbb{E}_{x}\left[\int_{0}^{\infty} \mathrm{e}^{-q t} \mathrm{~d} L_{t}^{\pi}-\Lambda \int_{0}^{\infty} \mathrm{e}^{-q t} \mathrm{~d} R_{t}^{\pi}\right],
$$

for any initial capital $x \geq 0$ and admissible policy $\pi=\left\{L^{\pi}, R^{\pi}\right\} \in \Theta$. Consider the strategy $\pi_{a, 0}=\left\{L^{a, 0}, R^{a, 0}\right\}$, which consists in setting reflecting barriers at $a$ and 0 , respectively. The controlled risk process $X^{\pi_{a, 0}}=X-L^{a, 0}+R^{a, 0}$ is a doubly reflected spectrally negative Lévy process and was studied by Avram et al. (2007). Intuitively, the process behaves similar to a Lévy process when it is inside $[0, a]$, but when it tries to cross above the level $a$ or below the level 0 it is forced to stay inside $[0, a]$. Using Theorem 1 from Avram et al. (2007), we have that for $a>0$ and $x \in[0, a]$,

$$
\begin{aligned}
\mathbb{E}_{x}\left[\int_{0}^{\infty} \mathrm{e}^{-q t} \mathrm{~d} L_{t}^{a, 0}\right] & =\frac{Z^{(q)}(x)}{q W^{(q)}(a)} \\
\mathbb{E}_{x}\left[\int_{0}^{\infty} \mathrm{e}^{-q t} \mathrm{~d} R_{t}^{a, 0}\right] & =\frac{Z^{(q)}(a)}{q W^{(q)}(a)} Z^{(q)}(x)-k^{(q)}(x) .
\end{aligned}
$$

Note that the expression in Equation (12) is finite under our assumption that $\psi^{\prime}(0+)>-\infty$. Using the expressions above, we can see that, for $\Lambda \geq 1$,

$$
v_{\Lambda}^{a}(x):=v_{\Lambda}^{\pi_{a, 0}}(x)= \begin{cases}Z^{(q)}(x) \zeta_{\Lambda}(a)+\Lambda k^{(q)}(x), & \text { if } 0 \leq x \leq a, \\ x-a+v_{\Lambda}^{a}(a), & \text { if } x>a,\end{cases}
$$

where

$$
\zeta_{\Lambda}(a):=\frac{1-\Lambda Z^{(q)}(a)}{q W^{(q)}(a)}, \quad a>0 .
$$

Equation (13) suggests that, to find the best barrier strategy we should maximize the function $\zeta_{\Lambda}$. Thus, we can define the candidate for the optimal barrier by

$$
a_{\Lambda}=\sup \left\{a \geq 0: \zeta_{\Lambda}(a) \geq \zeta_{\Lambda}(x), \text { for all } x \geq 0\right\} .
$$

Remark 6. Note that $\zeta_{\Lambda}:(0, \infty) \longrightarrow(-\infty, 0)$ and satisfies

$$
\lim _{a \rightarrow 0} \zeta_{\Lambda}(a)=-\frac{\Lambda-1}{q W^{(q)}(0)} \text { and } \lim _{a \rightarrow \infty} \zeta_{\Lambda}(a)=-\frac{\Lambda}{\Phi(q)} .
$$


Here, in case that $X$ is of unbounded variation, the first equality is understood to be $-\infty$. The barrier level $a_{\Lambda}$, given in Equation (15), corresponds with the level defined in Avram et al. (2007). Using the definition of the function $H$, we have that

$$
\frac{\mathrm{d} \zeta_{\Lambda}(a)}{\mathrm{d} a}=\frac{\Lambda W^{(q) \prime}(a)}{q\left[W^{(q)}(a)\right]^{2}}(H(a)-1 / \Lambda) .
$$

Since $H$ is strictly decreasing, $\zeta_{\Lambda}$ has a unique maximum at $a_{\Lambda}$ that is either a critical point, which is a solution of $H(a)=\frac{1}{\Lambda}$, or 0 if the right-hand derivative of $\zeta_{\Lambda}$ is negative at 0 . Therefore, by Remark 5 ,

$$
a_{\Lambda}= \begin{cases}0, & \text { if } \sigma=0, \Pi(0, \infty)<\infty \text { and } \Lambda<1+\frac{q}{\Pi(0, \infty)} \\ H^{-1}(1 / \Lambda), & \text { otherwise. }\end{cases}
$$

In addition, note that $\zeta_{\Lambda}$ is strictly increasing on $\left(0, a_{\Lambda}\right)$ and strictly decreasing on $\left(a_{\Lambda}, \infty\right)$.

Hence, from Avram et al. (2007), we know that the value function in Equation (3) and the optimal strategy are given by $V_{\Lambda}:=V_{0, \Lambda}=v_{\Lambda}^{a_{\Lambda}}$ and $\pi_{0, a_{\Lambda}}$, where $v_{\Lambda}^{a_{\Lambda}}$ and $a_{\Lambda}$ are as in Equations (13) and (16), respectively.

Remark 7. Note that the optimal barrier $a_{\Lambda} \rightarrow \infty$ as $\Lambda \rightarrow \infty$.

\section{Capital Injection and Fixed Transaction Cost}

In this section, we solve the problem in Equation (3) in the presence of a fixed transaction cost $\delta>0$. We consider strategies where the capital injection policy is $R^{0}$, given in Equation (6), and the dividend strategy is the so-called reflected $\left(c_{1}, c_{2}\right)$-policy, defined below.

\subsection{Value Function of Reflected $\left(c_{1}, c_{2}\right)$-Policies}

Let $\left(c_{1}, c_{2}\right)$ be a pair such that $0 \leq c_{1}<c_{2}$. In this subsection, we define the reflected $\left(c_{1}, c_{2}\right)$-policy, denoted by $\pi_{\left(c_{1}, c_{2}\right), 0}$, and under which we construct the controlled process. Let $Y=X+R^{0}$ be the Lévy process reflected from below 0 , so we set

$$
X_{t}^{\left(c_{1}, c_{2}\right), 0}=Y_{t}, \quad \text { for } t \leq T_{1}^{c_{1}, c_{2}},
$$

where $T_{1}^{c_{1}, c_{2}}=\inf \left\{t>0: Y_{t}>c_{2}\right\}$. The process then jumps downward by $Y_{T_{1}} c_{1}, c_{2}-c_{1}$ so that $X_{T_{1}^{1}, c_{2}}^{\left(c_{1}, c_{2}\right), 0}=c_{1}$. Now, for $T_{1}^{c_{1}, c_{2}} \leq t<T_{2}^{c_{1}, c_{2}}=\inf \left\{t>T_{1}^{c_{1}, c_{2}}: X_{t}^{\left(c_{1}, c_{2}\right), 0}>c_{2}\right\}, X^{\left(c_{1}, c_{2}\right), 0}$ is the reflected process from below at 0 of $X_{t}+\left(c_{1}-X_{T_{1}, c_{2}}\right)$, and $X_{T_{2}^{c_{1}, c_{2}}}^{\left(c_{1}, c_{2}\right), 0}=c_{1}$. By repeating this procedure, we can construct the process inductively. The process $X^{\left(c_{1}, c_{2}\right), 0}$ clearly admits the decomposition

$$
X_{t}^{\left(c_{1}, c_{2}\right), 0}=X_{t}-L_{t}^{\left(c_{1}, c_{2}\right), 0}+R_{t}^{\left(c_{1}, c_{2}\right), 0}, \quad t \geq 0,
$$

where $L^{\left(c_{1}, c_{2}\right), 0}$ and $R^{\left(c_{1}, c_{2}\right), 0}$ are the cumulative amounts of dividend payments and capital injection, respectively.

Let us compute the expected NPV of dividends with transaction costs for this strategy. For this purpose, we denote

$$
f_{c_{1}, c_{2}}(x)=\mathbb{E}_{x}\left[\int_{0}^{\infty} \mathrm{e}^{-q t} \mathrm{~d}\left(L_{t}^{\left(c_{1}, c_{2}\right), 0}-\delta \sum_{0 \leq s \leq t} \mathbf{1}_{\left\{\Delta L_{s}^{\left(c_{1}, c_{2}\right), 0}>0\right\}}\right)\right] .
$$


If $x<c_{2}$, by the Strong Markov Property and Equation (8), we obtain that

$$
f_{c_{1}, c_{2}}(x)=\mathbb{E}_{x}\left[\mathrm{e}^{-q T_{1}^{c_{1}, c_{2}}}\right] f_{c_{1}, c_{2}}\left(c_{2}\right)=\frac{Z^{(q)}(x)}{Z^{(q)}\left(c_{2}\right)} f_{c_{1}, c_{2}}\left(c_{2}\right) .
$$

When $x \geq c_{2}$, an amount $x-c_{1}$ is paid as dividends and a transaction cost $\delta$ is incurred immediately, so by using Equation (17) we obtain

$$
f_{c_{1}, c_{2}}(x)=x-c_{1}-\delta+f_{c_{1}, c_{2}}\left(c_{1}\right)=x-c_{1}-\delta+\frac{Z^{(q)}\left(c_{1}\right)}{Z^{(q)}\left(c_{2}\right)} f_{c_{1}, c_{2}}\left(c_{2}\right) .
$$

Hence, taking $x=c_{2}$, and solving for $f_{c_{1}, c_{2}}\left(c_{2}\right)$ we get

$$
f_{c_{1}, c_{2}}\left(c_{2}\right)=\left(c_{2}-c_{1}-\delta\right) \frac{Z^{(q)}\left(c_{2}\right)}{Z^{(q)}\left(c_{2}\right)-Z^{(q)}\left(c_{1}\right)} .
$$

Using the aforementioned expression in Equation (17), we have for $x<c_{2}$,

$$
f_{c_{1}, c_{2}}(x)=\left(c_{2}-c_{1}-\delta\right) \frac{Z^{(q)}(x)}{Z^{(q)}\left(c_{2}\right)-Z^{(q)}\left(c_{1}\right)} .
$$

Now, let us calculate the expected NPV of the injected capital denoted by

$$
g_{c_{1}, c_{2}}(x)=\mathbb{E}_{x}\left[\int_{0}^{\infty} \mathrm{e}^{-q t} \mathrm{~d} R_{t}^{\left(c_{1}, c_{2}\right), 0}\right] .
$$

Again, by the Strong Markov Property, noting that $T_{1}^{c_{1}, c_{2}}=\inf \left\{t>0: Y_{t} \in\left(c_{2}, \infty\right)\right\}$ and Equations (8)-(9), we have for $x \geq 0$

$$
\begin{aligned}
g_{c_{1}, c_{2}}(x) & =\mathbb{E}_{x}\left[\int_{\left[0, T_{1}^{c_{1}, c_{2}}\right]} \mathrm{e}^{-q t} \mathrm{~d} R_{t}^{0}\right]+\mathbb{E}_{x}\left[\mathrm{e}^{-q T_{1}^{c_{1}, c_{2}}}\right] g_{c_{1}, c_{2}}\left(c_{1}\right) \\
& =-k^{(q)}(x)+k^{(q)}\left(c_{2}\right) \frac{Z^{(q)}(x)}{Z^{(q)}\left(c_{2}\right)}+\frac{Z^{(q)}(x)}{Z^{(q)}\left(c_{2}\right)} g_{c_{1}, c_{2}}\left(c_{1}\right) .
\end{aligned}
$$

Thus, setting $x=c_{1}$ and solving for $g_{c_{1}, c_{2}}\left(c_{1}\right)$, we obtain

$$
\begin{aligned}
g_{c_{1}, c_{2}}\left(c_{1}\right) & =\left(-k^{(q)}\left(c_{1}\right)+k^{(q)}\left(c_{2}\right) \frac{Z^{(q)}\left(c_{1}\right)}{Z^{(q)}\left(c_{2}\right)}\right) \frac{Z^{(q)}\left(c_{2}\right)}{Z^{(q)}\left(c_{2}\right)-Z^{(q)}\left(c_{1}\right)} \\
& =\left(-\bar{Z}^{(q)}\left(c_{1}\right)+\bar{Z}^{(q)}\left(c_{2}\right) \frac{Z^{(q)}\left(c_{1}\right)}{Z^{(q)}\left(c_{2}\right)}\right) \frac{Z^{(q)}\left(c_{2}\right)}{Z^{(q)}\left(c_{2}\right)-Z^{(q)}\left(c_{1}\right)}-\frac{\psi^{\prime}(0+)}{q} .
\end{aligned}
$$

Putting the pieces together, we obtain

$$
\begin{aligned}
g_{c_{1}, c_{2}}(x)= & -k^{(q)}(x)+k^{(q)}\left(c_{2}\right) \frac{Z^{(q)}(x)}{Z^{(q)}\left(c_{2}\right)} \\
& +\left(\left(-\bar{Z}^{(q)}\left(c_{1}\right)+\bar{Z}^{(q)}\left(c_{2}\right) \frac{Z^{(q)}\left(c_{1}\right)}{Z^{(q)}\left(c_{2}\right)}\right) \frac{Z^{(q)}\left(c_{2}\right)}{Z^{(q)}\left(c_{2}\right)-Z^{(q)}\left(c_{1}\right)}-\frac{\psi^{\prime}(0+)}{q}\right) \frac{Z^{(q)}(x)}{Z^{(q)}\left(c_{2}\right)} \\
= & -k^{(q)}(x)+\bar{Z}^{(q)}\left(c_{2}\right) \frac{Z^{(q)}(x)}{Z^{(q)}\left(c_{2}\right)}+\left(-\bar{Z}^{(q)}\left(c_{1}\right)+\bar{Z}^{(q)}\left(c_{2}\right) \frac{Z^{(q)}\left(c_{1}\right)}{Z^{(q)}\left(c_{2}\right)}\right) \frac{Z^{(q)}(x)}{Z^{(q)}\left(c_{2}\right)-Z^{(q)}\left(c_{1}\right)} \\
= & Z^{(q)}(x)\left(\frac{\bar{Z}^{(q)}\left(c_{2}\right)-\bar{Z}^{(q)}\left(c_{1}\right)}{Z^{(q)}\left(c_{2}\right)-Z^{(q)}\left(c_{1}\right)}\right)-k^{(q)}(x) .
\end{aligned}
$$


Hence, we have the following result.

Lemma 1. The expected NPV associated with a reflected $\left(c_{1}, c_{2}\right)$-policy is given by

$$
v_{\delta, \Lambda}^{c_{1}, c_{2}}(x):=v_{\delta, \Lambda}^{\pi_{\left(c_{1}, c_{2}\right), 0}}(x)= \begin{cases}Z^{(q)}(x) G_{\Lambda}\left(c_{1}, c_{2}\right)+\Lambda k^{(q)}(x), & \text { if } x \leq c_{2} \\ x-c_{1}-\delta+v_{\delta, \Lambda}^{c_{1}, c_{2}}\left(c_{1}\right), & \text { if } x>c_{2}\end{cases}
$$

where

$$
G_{\Lambda}\left(c_{1}, c_{2}\right):=\frac{c_{2}-c_{1}-\delta-\Lambda\left(\bar{Z}^{(q)}\left(c_{2}\right)-\bar{Z}^{(q)}\left(c_{1}\right)\right)}{Z^{(q)}\left(c_{2}\right)-Z^{(q)}\left(c_{1}\right)}, \text { for all } c_{2}>c_{1} \geq 0 .
$$

Remark 8. Note that $G_{\Lambda}$ is $C^{2}$ on $\mathcal{A}:=\left\{\left(c_{1}, c_{2}\right) \in \mathbb{R}_{+}^{2}: c_{1}<c_{2}\right\}$, and

$$
\begin{aligned}
\lim _{c_{2} \downarrow c_{1}} G_{\Lambda}\left(c_{1}, c_{2}\right) & =-\infty, \text { for } c_{1} \geq 0 \text { fixed, } \\
\lim _{\left|c_{1}\right|+\left|c_{2}\right| \rightarrow \infty} G_{\Lambda}\left(c_{1}, c_{2}\right) & =\lim _{c_{2} \rightarrow \infty} G_{\Lambda}\left(c_{1}, c_{2}\right)=-\frac{\Lambda}{\Phi(q)} .
\end{aligned}
$$

\subsection{Choice of Optimal Thresholds}

To choose the optimal thresholds among reflected policies, we maximize the function $G_{\Lambda}$.

Proposition 1. The function $G_{\Lambda}$, defined in Equation (19), attains its maximum on $\mathcal{A}$.

Proof. Let $c_{1} \geq 0$ be fixed. The first derivative of $G_{\Lambda}$ with respect to $c_{2}$ is given by

$$
\partial_{\mathcal{c}_{2}} G_{\Lambda}\left(c_{1}, c_{2}\right)=\frac{q F_{\Lambda}\left(c_{1}, c_{2}\right) W^{(q)}\left(c_{2}\right)}{\left(Z^{(q)}\left(c_{2}\right)-Z^{(q)}\left(c_{1}\right)\right)^{2}},
$$

where

$$
\begin{aligned}
F_{\Lambda}\left(c_{1}, c_{2}\right):= & \frac{\left(Z^{(q)}\left(c_{2}\right)-Z^{(q)}\left(c_{1}\right)\right)}{q W^{(q)}\left(c_{2}\right)}\left(1-\Lambda Z^{(q)}\left(c_{2}\right)\right)-\left(c_{2}-c_{1}-\delta-\Lambda\left(\bar{Z}^{(q)}\left(c_{2}\right)-\bar{Z}^{(q)}\left(c_{1}\right)\right)\right) \\
= & -\Lambda\left[\frac{\left(Z^{(q)}\left(c_{2}\right)\right)^{2}}{q W^{(q)}\left(c_{2}\right)}-\bar{Z}^{(q)}\left(c_{2}\right)-\left(\frac{\left(Z^{(q)}\left(c_{2}\right)\right)}{q W^{(q)}\left(c_{2}\right)} Z^{(q)}\left(c_{1}\right)-\bar{Z}^{(q)}\left(c_{1}\right)\right)\right] \\
& +\frac{Z^{(q)}\left(c_{2}\right)-Z^{(q)}\left(c_{1}\right)}{q W^{(q)}\left(c_{2}\right)}-\left(c_{2}-c_{1}-\delta\right) .
\end{aligned}
$$

On the other hand, taking $a=c_{2}$ in Equation (12), we see

$$
\frac{\left[Z^{(q)}\left(c_{2}\right)\right]^{2}}{q W^{(q)}\left(c_{2}\right)}-k^{(q)}\left(c_{2}\right) \geq 0 \quad \text { and } \quad \frac{Z^{(q)}\left(c_{2}\right)}{q W^{(q)}\left(c_{2}\right)} Z^{(q)}\left(c_{1}\right)-k^{(q)}\left(c_{1}\right) \geq 0 .
$$

Then, using Equation (10), we have

$$
F_{\Lambda}\left(c_{1}, c_{2}\right)<\frac{Z^{(q)}\left(c_{2}\right)}{q W^{(q)}\left(c_{2}\right)}+\Lambda\left[\frac{Z^{(q)}\left(c_{2}\right)}{q W^{(q)}\left(c_{2}\right)} Z^{(q)}\left(c_{1}\right)-k^{(q)}\left(c_{1}\right)\right]-\left(c_{2}-c_{1}-\delta\right) .
$$

Therefore, since $\lim _{c_{2} \rightarrow \infty} \frac{Z^{(q)}\left(c_{2}\right)}{q W^{(q)}\left(c_{2}\right)}=\frac{1}{\Phi(q)}$ (see Remark 3), the right-hand side of the aforementioned inequality goes to $-\infty$ as $c_{2}$ goes to $\infty$, which implies

$$
\partial_{c_{2}} G_{\Lambda}\left(c_{1}, c_{2}\right)<0, \quad \text { for } c_{2} \text { large enough. }
$$


From here and Remark 8, we obtain that there exists $c^{*} \in\left(c_{1}, \infty\right)$ (that depends on $\left.c_{1}\right)$ such that

$$
G_{\Lambda}\left(c_{1}, c_{2}\right) \leq G_{\Lambda}\left(c_{1}, c^{*}\right), \quad \text { for all } c_{2}>c_{1} .
$$

Taking $d^{*}\left(c_{1}\right):=\sup \left\{c^{*}>c_{1}: G_{\Lambda}\left(c_{1}, c_{2}\right) \leq G_{\Lambda}\left(c_{1}, c^{*}\right)\right.$ for all $\left.c_{2}>c_{1}\right\}$, with $c_{1} \geq 0$, we see $d^{*}\left(c_{1}\right)<\infty$ for each $c_{1} \geq 0$, since Equation (23) holds. From Equation (20) and the fact that $\partial_{c_{2}} G_{\Lambda}\left(c_{1}, d^{*}\left(c_{1}\right)\right)=0$, it follows that $F_{\Lambda}\left(c_{1}, d^{*}\left(c_{1}\right)\right)=0$ for $c_{1} \geq 0$. Then, by the definitions of $F_{\Lambda}$ and $\zeta_{\Lambda}$-see Equations (21) and (14), respectively-we get

$$
G_{\Lambda}\left(c_{1}, d^{*}\left(c_{1}\right)\right)=\frac{d^{*}\left(c_{1}\right)-c_{1}-\delta-\Lambda\left(\bar{Z}^{(q)}\left(d^{*}\left(c_{1}\right)\right)-\bar{Z}^{(q)}\left(c_{1}\right)\right)}{Z^{(q)}\left(d^{*}\left(c_{1}\right)\right)-Z^{(q)}\left(c_{1}\right)}=\zeta_{\Lambda}\left(d^{*}\left(c_{1}\right)\right), \quad \text { for each } c_{1} \geq 0 .
$$

Now, let us take $\bar{c}_{1}>a_{\Lambda}$ (where $a_{\Lambda}$ is defined in Equation (14)). Then, using the fact that $\zeta_{\Lambda}$ is strictly decreasing in $\left(a_{\Lambda}, \infty\right)$ (see Remark 6), we have that for any $c_{2}>c_{1}>d^{*}\left(\bar{c}_{1}\right)$ it holds that $d^{*}\left(\bar{c}_{1}\right)<d^{*}\left(c_{1}\right)$ and

$$
G_{\Lambda}\left(c_{1}, c_{2}\right) \leq G_{\Lambda}\left(c_{1}, d^{*}\left(c_{1}\right)\right)=\zeta_{\Lambda}\left(d^{*}\left(c_{1}\right)\right)<\zeta_{\Lambda}\left(d^{*}\left(\bar{c}_{1}\right)\right)=G_{\Lambda}\left(\bar{c}_{1}, d^{*}\left(\bar{c}_{1}\right)\right) .
$$

This implies that the maximum of the function $G_{\Lambda}$ has to be achieved on the set

$$
\left\{\left(c_{1}, c_{2}\right) \in \mathbb{R}_{+}^{2}: c_{1}<c_{2} \text { and } c_{1} \in\left[0, \bar{c}_{1}\right]\right\} .
$$

Finally, from Equation (22), we obtain

$$
F_{\Lambda}\left(c_{1}, c_{2}\right)<\frac{Z^{(q)}\left(c_{2}\right)}{q W^{(q)}\left(c_{2}\right)}+\Lambda \sup _{c_{1} \in\left[0, \bar{c}_{1}\right]}\left[\frac{Z^{(q)}\left(c_{2}\right)}{q W^{(q)}\left(c_{2}\right)} Z^{(q)}\left(c_{1}\right)-k^{(q)}\left(c_{1}\right)\right]-\left(c_{2}-\bar{c}_{1}-\delta\right), \quad \text { for } c_{1} \in\left[0, \bar{c}_{1}\right] .
$$

Hence, for any $c_{1} \in\left[0, \bar{c}_{1}\right]$, we can find $\bar{c}_{2}>\bar{c}_{1}$ such that

$$
\partial_{c_{2}} G_{\Lambda}\left(c_{1}, c_{2}\right)\left(c_{1}, c_{2}\right)<0, \quad \text { for any } 0 \leq c_{1} \leq \bar{c}_{1} \text { and } 0 \leq c_{2} \leq \bar{c}_{2} .
$$

Therefore, the function $G_{\Lambda}$ attains its maximum on the set

$$
\left\{\left(c_{1}, c_{2}\right) \in\left[0, \bar{c}_{1}\right] \times\left[0, \bar{c}_{2}\right]: c_{1}<c_{2}\right\} \subset \mathcal{A} .
$$

Note that by Proposition 1 the set $\mathcal{B} \subset \mathcal{A}$ defined as

$$
\mathcal{B}:=\left\{\left(c_{1}^{*}, c_{2}^{*}\right) \in \mathcal{A}: G_{\Lambda}\left(c_{1}^{*}, c_{2}^{*}\right) \geq G_{\Lambda}\left(c_{1}, c_{2}\right) \text { for all }\left(c_{1}, c_{2}\right) \in \mathcal{A}\right\},
$$

is not empty. Moreover, since $G_{\Lambda} \in \mathrm{C}^{1}(\mathcal{A})$ and using Equation (14), it follows that

$$
\partial_{c_{1}} G_{\Lambda}\left(c_{1}^{*}, c_{2}^{*}\right)=\frac{q W^{(q)}\left(c_{1}^{*}\right)}{Z^{(q)}\left(c_{2}^{*}\right)-Z^{(q)}\left(c_{1}^{*}\right)}\left(G_{\Lambda}\left(c_{1}^{*}, c_{2}^{*}\right)-\zeta_{\Lambda}\left(c_{1}^{*}\right)\right) \leq 0, \text { for }\left(c_{1}^{*}, c_{2}^{*}\right) \in \mathcal{B},
$$

with equality if $c_{1}>0$, and

$$
\partial_{c_{2}} G_{\Lambda}\left(c_{1}^{*}, c_{2}^{*}\right)=-\frac{q W^{(q)}\left(c_{2}^{*}\right)}{Z^{(q)}\left(c_{2}^{*}\right)-Z^{(q)}\left(c_{1}^{*}\right)}\left(G_{\Lambda}\left(c_{1}^{*}, c_{2}^{*}\right)-\zeta_{\Lambda}\left(c_{2}^{*}\right)\right)=0, \quad \text { for }\left(c_{1}^{*}, c_{2}^{*}\right) \in \mathcal{B} .
$$


Proposition 2. There exists a unique pair $\left(c_{1}^{\Lambda}, c_{2}^{\Lambda}\right)$ in $\mathcal{B}$. Furthermore, $0 \leq c_{1}^{\Lambda} \leq a_{\Lambda}<c_{2}^{\Lambda}<\infty$, with $a_{\Lambda}$ defined in Equation (16), and the value function associated with the $\left(c_{1}^{\Lambda}, c_{2}^{\Lambda}\right)$-policy is

$$
v_{\delta, \Lambda}^{c_{1}^{\Lambda}, c_{2}^{\Lambda}}(x)= \begin{cases}Z^{(q)}(x) \zeta_{\Lambda}\left(c_{2}^{\Lambda}\right)+\Lambda k^{(q)}(x), & \text { if } x \leq c_{2}^{\Lambda}, \\ x-c_{2}^{\Lambda}+v_{\delta, \Lambda}^{c_{1}^{\Lambda}, c_{2}^{\Lambda}}\left(c_{2}^{\Lambda}\right), & \text { if } x>c_{2}^{\Lambda} .\end{cases}
$$

Proof. Let $M$ be the maximum value of $G_{\Lambda}$ in $\mathcal{B}$; therefore, for any $\left(c_{1}^{*}, c_{2}^{*}\right) \in \mathcal{B}$, we have that $\zeta_{\Lambda}\left(c_{2}^{*}\right)=M$ by Equation (25). From Remark 6 , we know that $\zeta_{\Lambda}$ is strictly increasing on $\left(0, a_{\Lambda}\right)$ and strictly decreasing on $\left(a_{\Lambda}, \infty\right)$. If $\zeta_{\Lambda}(0) \geq M, \zeta_{\Lambda}$ attains $M$ at a unique $c_{2}^{\Lambda}>a_{\Lambda}$ and therefore $\left(0, c_{2}^{\Lambda}\right)$ is the only point that satisfies Equation (24). On the other hand, if $\zeta_{\Lambda}(0)<M, \zeta_{\Lambda}$ can only attain the value $M$ at a unique $c_{1}^{\Lambda}<a_{\Lambda}$ and a unique $c_{2}^{\Lambda}>a_{\Lambda}$. Hence, $\left(c_{1}^{\Lambda}, c_{2}^{\Lambda}\right)$ is the only point that satisfies Equations (24) and (25), that is, the only existing point in $\mathcal{B}$. Now, from Lemma 1 and using that $G_{\Lambda}\left(c_{1}^{\Lambda}, c_{2}^{\Lambda}\right)=\zeta_{\Lambda}\left(c_{2}^{\Lambda}\right)$, we obtain the first part of Equation (26). For the second part, let $x>c_{2}^{\Lambda}$, then

$$
v_{\delta, \Lambda}^{c_{1}^{\Lambda}, c_{2}^{\Lambda}}(x)=x-c_{1}^{\Lambda}-\delta+v_{\delta, \Lambda}^{c_{1}^{\Lambda}, c_{2}^{\Lambda}}\left(c_{1}^{\Lambda}\right)=x-c_{2}^{\Lambda}+c_{2}^{\Lambda}-c_{1}^{\Lambda}-\delta+v_{\delta, \Lambda}^{c_{1}^{\Lambda}, c_{2}^{\Lambda}}\left(c_{1}^{\Lambda}\right)=x-c_{2}^{\Lambda}+v_{\delta, \Lambda}^{c_{1}^{\Lambda}, c_{2}^{\Lambda}}\left(c_{2}^{\Lambda}\right) .
$$

The following properties of $v_{\delta, \Lambda}^{c_{1}^{\Lambda}, c_{2}^{\Lambda}}$ are used below in the verification theorem.

Remark 9. From Equations (10) and (26), we note

$$
v_{\delta, \Lambda}^{c_{1}^{\Lambda}, c_{2}^{\Lambda}}(x) \geq \frac{\Lambda \psi^{\prime}(0+)}{q}+Z^{(q)}\left(c_{2}^{\Lambda}\right) \zeta\left(c_{2}^{\Lambda}\right), \text { for } x>0 .
$$

Remark 10 (Continuity/smoothness at zero). Note that for $x<0, v_{\delta, \Lambda}^{c_{1}^{\Lambda}, c_{2}^{\Lambda}}(x)=v_{\delta, \Lambda}^{c_{1}^{\Lambda}, c_{2}^{\Lambda}}(0)+\Lambda x$. Therefore,

(i) $v_{\delta, \Lambda}^{c_{1}^{\Lambda}, c_{2}^{\Lambda}}$ is continuous at zero.

(ii) For the case of unbounded variation, we have that

$$
v_{\delta, \Lambda}^{c_{1}^{\Lambda}, c_{2}^{\Lambda \prime}}(0+)=q W^{(q)}(0+) \zeta_{\Lambda}\left(c_{2}^{\Lambda}\right)+\Lambda=\Lambda=v_{\delta, \Lambda}^{c_{1}^{\Lambda}, c_{2}^{\Lambda \prime}}(0-)
$$

\subsection{Verification}

Let us denote by $v_{\delta, \Lambda}$ the function given in Equation (26), which is the optimal value function among reflected policies. We now prove some properties of this function.

Lemma 2. The function $v_{\delta, \Lambda}$ is $C^{2}\left((0, \infty) \backslash\left\{c_{2}^{\Lambda}\right\}\right)$ and $C^{1}(0, \infty)$.

Proof. By Assumption 1, we have that, for each $q \geq 0$, the function $W^{(q)}$ is continuously differentiable on $(0, \infty)$. This implies, by Equation (26), that $v_{\delta, \Lambda}$ is $C^{2}\left((0, \infty) \backslash\left\{c_{2}^{\Lambda}\right\}\right)$. On the other hand, using Equation (26), we have that for $x \leq c_{2}^{\Lambda}$,

$$
v_{\delta, \Lambda}^{\prime}(x)=q W^{(q)}(x) \zeta_{\Lambda}\left(c_{2}^{\Lambda}\right)+\Lambda Z^{(q)}(x)=q W^{(q)}(x)\left(\frac{1-\Lambda Z^{(q)}\left(c_{2}^{\Lambda}\right)}{q W^{(q)}\left(c_{2}^{\Lambda}\right)}\right)+\Lambda Z^{(q)}(x) .
$$

This implies that $v_{\delta, \Lambda}^{\prime}\left(c_{2}^{\Lambda}-\right)=1$. For $x>c_{2}^{\Lambda}$, we obtain by Equation (26) that

$$
v_{\delta, \Lambda}^{\prime}\left(c_{2}^{\Lambda}+\right)=1=v_{\delta, \Lambda}^{\prime}\left(c_{2}^{\Lambda}-\right),
$$

which implies the result. 
Let $\mathcal{L}$ be the operator defined as follows,

$$
\mathcal{L} F(x):=\gamma F^{\prime}(x)+\frac{\sigma^{2}}{2} F^{\prime \prime}(x)+\int_{(0, \infty)}\left(F(x-z)-F(x)+F^{\prime}(x) z \mathbf{1}_{\{0<z \leq 1\}}\right) \Pi(\mathrm{d} z), \quad x>0,
$$

where $x \in \mathbb{R}$ and $F$ is a function on $\mathbb{R}$ such that $\mathcal{L} F(x)$ is well defined.

\section{Proposition 3.}

1. $(\mathcal{L}-q) v_{\delta, \Lambda}(x)=0$ for $x<c_{2}^{\Lambda}$.

2. $(\mathcal{L}-q) v_{\delta, \Lambda}(x) \leq 0$ for $x>c_{2}^{\Lambda}$.

\section{Proof.}

1. By the proof of Theorem 2.1 in Bayraktar et al. (2014a), we have that for $0<x<c_{2}^{\Lambda}$,

$$
(\mathcal{L}-q)\left(\bar{Z}^{(q)}(x)+\frac{\psi^{\prime}(0+)}{q}\right)=0 \quad \text { and } \quad(\mathcal{L}-q) Z^{(q)}(x)=0 .
$$

This implies that for $0<x<c_{2}^{\Lambda}$,

$$
(\mathcal{L}-q) v_{\delta, \Lambda}(x)=0
$$

2. We note that $v_{\delta, \Lambda}(y)=u_{\Lambda}^{c_{2}^{\Lambda}}(y)$ for all $y \geq 0$, where $u_{\Lambda}^{a}$ is the barrier strategy at the level $a$ for the dividend problem with capital injection given by Equation (13). Therefore,

(i) If we take $y \leq x$, and $c_{2}^{\Lambda} \leq x$, we obtain

$$
u_{\Lambda}^{x}(y)=Z^{(q)}(y) \zeta_{\Lambda}(x)+\Lambda\left(\bar{Z}^{(q)}(y)+\frac{\psi^{\prime}(0+)}{q}\right) .
$$

Recall the functions $\zeta_{\Lambda}$ and $H$ are as in Equations (7) and (14), respectively. Then,

$$
\begin{aligned}
\lim _{y \uparrow x} \frac{\mathrm{d}^{2} u_{\Lambda}^{x}}{\mathrm{~d} y^{2}}(y) & =\Lambda q W^{(q)}(x)+q W^{(q) \prime}(x) \zeta_{\Lambda}(x) \\
& =\frac{W^{(q) \prime}(x)}{W^{(q)}(x)}\left(1-\Lambda\left(Z^{(q)}(x)-q W^{(q)}(x) \frac{W^{(q)}(x)}{W^{(q) \prime}(x)}\right)\right) \\
& =\frac{W^{(q) \prime}(x)}{W^{(q)}(x)}(1-\Lambda H(x)) \\
& =-q W^{(q)}(x) \zeta_{\Lambda}^{\prime}(x) .
\end{aligned}
$$

By Proposition 2, we know that $a_{\Lambda}<c_{2}^{\Lambda} \leq x$. Then, $\lim _{y \uparrow x} \frac{\mathrm{d}^{2} u_{\Lambda}^{x}}{\mathrm{~d} y^{2}}(y) \geq 0=\frac{\mathrm{d}^{2} u_{\Lambda}^{c_{2}^{\Lambda}}}{\mathrm{d} x^{2}}(x)$, since $\zeta_{\Lambda}^{\prime}(x)<0$ by Remark 6 .

(ii) We have for $y \in\left[0, c_{2}^{\Lambda}\right]$,

$$
\frac{\mathrm{d} u_{\Lambda}^{c_{2}^{\Lambda}}}{\mathrm{d} y}(y)=\Lambda Z^{(q)}(y)+q W^{(q)}(y) \zeta_{\Lambda}\left(c_{2}^{\Lambda}\right) \geq \Lambda Z^{(q)}(y)+q W^{(q)}(y) \zeta_{\Lambda}(x)=\frac{\mathrm{d} u_{\Lambda}^{x}}{\mathrm{~d} y}(y),
$$


which comes from the fact that for $x \geq c_{2}^{\Lambda}>a_{\Lambda}$, then $\zeta_{\Lambda}\left(c_{2}^{\Lambda}\right) \geq \zeta_{\Lambda}(x)$ by Remark 2 . On the other hand, for $y \in\left(c_{2}^{\Lambda}, x\right]$, we have, using the fact that $\zeta_{\Lambda}(y) \geq \zeta_{\Lambda}(x)$,

$$
\begin{aligned}
\frac{\mathrm{d} u_{\Lambda}^{x}}{\mathrm{~d} y}(y) & =\Lambda Z^{(q)}(y)+q W^{(q)}(y) \zeta_{\Lambda}(x) \\
& \leq \Lambda Z^{(q)}(y)+q W^{(q)}(y) \zeta_{\Lambda}(y) \\
& =\Lambda Z^{(q)}(y)+q W^{(q)}(y) \frac{1-\Lambda Z^{(q)}(y)}{q W^{(q)}(y)}=1=\frac{\mathrm{d} u_{\Lambda}^{c_{2}^{\Lambda}}}{\mathrm{d} y}(y) .
\end{aligned}
$$

(iii) We note that

$$
\begin{aligned}
u_{\Lambda}^{x}\left(c_{2}^{\Lambda}\right) & =\Lambda\left(\bar{Z}^{(q)}\left(c_{2}^{\Lambda}\right)+\frac{\psi^{\prime}(0+)}{q}\right)+Z^{(q)}\left(c_{2}^{\Lambda}\right) \zeta_{\Lambda}(x) \\
& \leq \Lambda\left(\bar{Z}^{(q)}\left(c_{2}^{\Lambda}\right)+\frac{\psi^{\prime}(0+)}{q}\right)+Z^{(q)}\left(c_{2}^{\Lambda}\right) \zeta_{\Lambda}\left(c_{2}^{\Lambda}\right)=u_{\Lambda}^{c_{2}^{\Lambda}}\left(c_{2}^{\Lambda}\right) .
\end{aligned}
$$

This and Point (ii) imply that $\left(u_{\Lambda}^{c_{2}^{\Lambda}}-u_{\Lambda}^{x}\right)(x) \geq 0$.

(iv) We have

$$
\frac{\mathrm{d} u_{\Lambda}^{c_{2}^{\Lambda}}}{\mathrm{d} x}(x)=1=\lim _{y \rightarrow x} \frac{\mathrm{d} u_{\Lambda}^{x}}{\mathrm{~d} y}(y) .
$$

Thus, by similar arguments to those in the proof of Theorem 2 in Loeffen (2008), we obtain the result.

\section{Lemma 3.}

1. For $x>0$, we have that $v_{\delta, \Lambda}^{\prime}(x) \leq \Lambda$.

2. For $x \geq y \geq 0$, we have that $v_{\delta, \Lambda}(x)-v_{\delta, \Lambda}(y) \geq x-y-\delta$.

Proof. 1. By Equation (5) together with Equation (26), we note that for $x \leq c_{2}^{\Lambda}$,

$$
\begin{aligned}
v_{\delta, \Lambda}^{\prime}(x) & =\Lambda\left(Z^{(q)}(x)-\frac{W^{(q)}(x)}{W^{(q)}\left(c_{2}^{\Lambda}\right)} Z^{(q)}\left(c_{2}^{\Lambda}\right)\right)+\frac{W^{(q)}(x)}{W^{(q)}\left(c_{2}^{\Lambda}\right)} \\
& =\Lambda \mathbb{E}_{x}\left[\mathrm{e}^{-q \tau_{0}^{-}} \mathbf{1}_{\left\{\tau_{0}^{-}<\tau_{c_{2}^{\Lambda}}^{+}\right\}}\right]+\mathbb{E}_{x}\left[\mathrm{e}^{-q \tau_{c_{2}^{\Lambda}}^{+}} \mathbf{1}_{\left\{\tau_{c_{2}^{\Lambda}}^{+}<\tau_{0}^{-}\right\}}\right] \\
& \leq \Lambda\left(\mathbb{E}_{x}\left[\mathrm{e}^{-q \tau_{0}^{-}} \mathbf{1}_{\left\{\tau_{0}^{-}<\tau_{c_{2}^{\Lambda}}^{+}\right\}}\right]+\mathbb{E}_{x}\left[\mathrm{e}^{-q \tau_{c_{2}^{\Lambda}}^{+}} \mathbf{1}_{\left\{\tau_{c_{2}^{\Lambda}}^{+}<\tau_{0}^{-}\right\}}\right]\right) \\
& \leq \Lambda\left(\mathbb{P}_{x}\left[\tau_{0}^{-}<\tau_{c_{2}^{\Lambda}}^{+}\right]+\mathbb{P}_{x}\left[\tau_{c_{2}^{+}}^{+}<\tau_{0}^{-}\right]\right)=\Lambda .
\end{aligned}
$$

On the other hand, $v_{\delta, \Lambda}^{\prime}(x)=1 \leq \Lambda$ for $x>c_{2}^{\Lambda}$.

2. Let us consider $c_{2}^{\Lambda} \geq x \geq y$. We note that

$$
\begin{aligned}
v_{\delta, \Lambda}(x)-v_{\delta, \Lambda}(y) & =\Lambda\left(\bar{Z}^{(q)}(x)-\bar{Z}^{(q)}(y)\right)+\left(Z^{(q)}(x)-Z^{(q)}(y)\right) \zeta_{\Lambda}\left(c_{2}^{\Lambda}\right) \\
& =\Lambda\left(\bar{Z}^{(q)}(x)-\bar{Z}^{(q)}(y)\right)+\left(Z^{(q)}(x)-Z^{(q)}(y)\right) G_{\Lambda}\left(c_{1}^{\Lambda}, c_{2}^{\Lambda}\right)
\end{aligned}
$$




$$
\begin{aligned}
& \geq \Lambda\left(\bar{Z}^{(q)}(x)-\bar{Z}^{(q)}(y)\right)+\left(Z^{(q)}(x)-Z^{(q)}(y)\right) G_{\Lambda}(x, y) \\
& =\Lambda\left(\bar{Z}^{(q)}(x)-\bar{Z}^{(q)}(y)\right)+\left(Z^{(q)}(x)-Z^{(q)}(y)\right) \frac{x-y-\delta-\Lambda\left(\bar{Z}^{(q)}(x)-\bar{Z}^{(q)}(y)\right)}{Z^{(q)}(x)-Z^{(q)}(y)} \\
& =x-y-\delta .
\end{aligned}
$$

Now, suppose that $x \geq y \geq c_{2}^{\Lambda}$, then using Equation (26) we obtain

$$
v_{\delta, \Lambda}(x)-v_{\delta, \Lambda}(y)=x-y \geq x-y-\delta .
$$

Finally, for the case $x \geq c_{2}^{\Lambda} \geq y$, by Equation (27), we have

$$
v_{\delta, \Lambda}(x)-v_{\delta, \Lambda}(y)=x-c_{2}^{\Lambda}+v_{\delta, \Lambda}\left(c_{2}^{\Lambda}\right)-v_{\delta, \Lambda}(y) \geq x-c_{2}^{\Lambda}+\left(c_{2}^{\Lambda}-y-\delta\right)=x-y-\delta .
$$

Now, we proceed to the verification theorem that proves the optimality of the $\left(c_{1}^{\Lambda}, c_{2}^{\Lambda}\right)$-policy.

Theorem 1 (Verification Theorem). Let $V_{\delta, \Lambda}, v_{\delta, \Lambda}$ be as in Equations (3) and (26), respectively. Then, $v_{\delta, \Lambda}(x)=V_{\delta, \Lambda}(x)$ for all $x \geq 0$. Hence, the $\left(c_{1}^{\Lambda}, c_{2}^{\Lambda}\right)$-policy is optimal.

Proof. By the definition of $V_{\delta, \Lambda}, v_{\delta, \Lambda}(x) \leq V_{\delta, \Lambda}(x)$ for all $x \geq 0$. Let us verify that $v_{\delta, \Lambda}(x) \geq v_{\delta, \Lambda}^{\pi}(x)$ for all admissible $\pi \in \Theta_{\delta}$ and for all $x \geq 0$. Recall that $v_{\delta, \Lambda}^{\pi}$ is defined in Equation (2). Take $\pi=\left\{L^{\pi}, R^{\pi}\right\} \in$ $\Theta_{\delta}$ fixed and let $\left(T_{n}\right)_{n \in \mathbb{N}}$ be the sequence of stopping times where $T_{n}:=\inf \left\{t>0: X_{t}^{\pi}>n\right\}$. Since $X^{\pi}=X-L^{\pi}+R^{\pi}$, with $X$ being a spectrally negative Lévy process, it is a semi-martingale and $v_{\delta, \Lambda}$ is sufficiently smooth on $(0, \infty)$ by Lemma 2 , and continuous (respectively, continuously differentiable) at zero for the case of bounded variation (respectively, unbounded variation) by Remark 10, we can use the change of variables/Meyer-Itô's formula (cf. Theorems II.31 and II.32 of Protter (2005)) on the stopped process $\left(\mathrm{e}^{-q\left(t \wedge T_{n}\right)} v_{\delta, \Lambda}\left(X_{t \wedge T_{n}}^{\pi}\right) ; t \geq 0\right)$ to deduce under $\mathbb{P}_{x}$ that

$$
\begin{aligned}
\mathrm{e}^{-q\left(t \wedge T_{n}\right)} v_{\delta, \Lambda}\left(X_{t \wedge T_{n}}^{\pi}\right)-v_{\delta, \Lambda}(x)= & \int_{0}^{t \wedge T_{n}} \mathrm{e}^{-q s}(\mathcal{L}-q) v_{\delta, \Lambda}\left(X_{s-}^{\pi}\right) \mathrm{d} s+M_{t \wedge T_{n}}+J_{t \wedge T_{n}} \\
& +\int_{\left[0, t \wedge T_{n}\right]} \mathrm{e}^{-q s} v_{\delta, \Lambda}^{\prime}\left(X_{s-}^{\pi}\right) \mathrm{d} R_{s}^{\pi, c},
\end{aligned}
$$

where $M$ is a local martingale with $M_{0}=0, R^{\pi, c}$ is the continuous part of $R^{\pi}$, and $J$ is a jump process, which is given by

$$
\begin{aligned}
J_{t}= & \sum_{0 \leq s \leq t} \mathrm{e}^{-q s}\left(v_{\delta, \Lambda}\left(X_{s-}^{\pi}+\Delta\left[X+R^{\pi}\right]_{s}\right)-v_{\delta, \Lambda}\left(X_{s-}^{\pi}+\Delta X_{s}\right)\right) 1_{\left\{\Delta[X+R]_{s} \neq 0\right\}} \\
& +\sum_{0 \leq s \leq t} \mathrm{e}^{-q s}\left(v_{\delta, \Lambda}\left(X_{s-}^{\pi}+\Delta\left[X+R^{\pi}\right]_{s}-\Delta L_{s}^{\pi}\right)-v_{\delta, \Lambda}\left(X_{s-}^{\pi}+\Delta\left[X+R^{\pi}\right]_{s}\right)\right) 1_{\left\{\Delta L_{s}^{\pi} \neq 0\right\}}, \text { for } t \geq 0 .
\end{aligned}
$$

On the other hand, by Part (1) of Lemma 3, we obtain that

$$
\begin{aligned}
& \int_{\left[0, t \wedge T_{n}\right]} \mathrm{e}^{-q s} v_{\delta, \Lambda}^{\prime}\left(X_{s-}^{\pi}\right) \mathrm{d} R_{s}^{\pi, c} \\
& +\sum_{0 \leq s \leq t \wedge T_{n}} \mathrm{e}^{-q s}\left[v_{\delta, \Lambda}\left(X_{s-}^{\pi}+\Delta\left[X+R^{\pi}\right]_{s}\right)-v_{\delta, \Lambda}\left(X_{s-}^{\pi}+\Delta X_{s}\right)\right] 1_{\left\{\Delta\left[X+R^{\pi}\right]_{s} \neq 0\right\}} \\
& \leq \Lambda \int_{\left[0, t \wedge T_{n}\right]} \mathrm{e}^{-q s} \mathrm{~d} R_{s}^{\pi, c}+\Lambda \sum_{0 \leq s \leq t \wedge T_{n}} \mathrm{e}^{-q s} \Delta R_{s}^{\pi}=\Lambda \int_{\left[0, t \wedge T_{n}\right]} \mathrm{e}^{-q s} \mathrm{~d} R_{s}^{\pi} .
\end{aligned}
$$


Similarly, by Part (2) of Lemma 3,

$$
\begin{aligned}
\sum_{0 \leq s \leq t \wedge T_{n}} \mathrm{e}^{-q s}\left[v _ { \delta , \Lambda } \left(X_{s-}^{\pi}+\right.\right. & \left.\left.\Delta\left[X+R^{\pi}\right]_{s}-\Delta L_{s}^{\pi}\right)-v_{\delta, \Lambda}\left(X_{s-}^{\pi}+\Delta\left[X+R^{\pi}\right]_{s}\right)\right] 1_{\left\{\Delta L_{s}^{\pi} \neq 0\right\}} \\
& \leq-\sum_{0 \leq s \leq t \wedge T_{n}} \mathrm{e}^{-q s} \Delta L_{s}^{\pi}+\delta \sum_{0 \leq s \leq t \wedge T_{n}} \mathrm{e}^{-q s} 1_{\left\{\Delta L_{s}^{\pi}>0\right\}} \\
& =-\int_{\left[0, t \wedge T_{n}\right]} \mathrm{e}^{-q s} \mathrm{~d}\left(L_{s}^{\pi}-\delta \sum_{0 \leq u \leq s} 1_{\left\{\Delta L_{u}^{\pi}>0\right\}}\right) .
\end{aligned}
$$

Hence, from Equation (28), we derive that

$$
\begin{aligned}
v_{\delta, \Lambda}(x) \geq & -\int_{0}^{t \wedge T_{n}} \mathrm{e}^{-q s}(\mathcal{L}-q) v_{\delta, \Lambda}\left(X_{s-}^{\pi}\right) \mathrm{d} s-\Lambda \int_{\left[0, t \wedge T_{n}\right]} \mathrm{e}^{-q s} \mathrm{~d} R_{s}^{\pi} \\
& +\int_{\left[0, t \wedge T_{n}\right]} \mathrm{e}^{-q s} \mathrm{~d}\left(L_{s}^{\pi}-\delta \sum_{0 \leq u \leq s} 1_{\left\{\Delta L_{u}^{\pi}>0\right\}}\right)-M_{t \wedge T_{n}}+\mathrm{e}^{-q\left(t \wedge T_{n}\right)} v_{\delta, \Lambda}\left(X_{t \wedge T_{n}}^{\pi}\right) .
\end{aligned}
$$

Using Proposition 3 along with Point 3 in the proof of Lemma 6 in Loeffen (2009), and that $X_{s-}^{\pi} \geq 0$ a.s. for $s \geq 0$, we observe that

$$
\begin{aligned}
v_{\delta, \Lambda}(x) \geq & \int_{\left[0, t \wedge T_{n}\right]} \mathrm{e}^{-q s} \mathrm{~d}\left(L_{s}^{\pi}-\delta \sum_{0 \leq u \leq s} 1_{\left\{\Delta L_{u}^{\pi}>0\right\}}\right)-\Lambda \int_{\left[0, t \wedge T_{n}\right]} \mathrm{d} R_{s}^{\pi}-M_{t \wedge T_{n}}+\mathrm{e}^{-q\left(t \wedge T_{n}\right)} v_{\delta, \Lambda}\left(X_{t \wedge T_{n}}^{\pi}\right) \\
\geq & \int_{\left[0, t \wedge T_{n}\right]} \mathrm{e}^{-q s} \mathrm{~d}\left(L_{s}^{\pi}-\delta \sum_{0 \leq u \leq s} 1_{\left\{\Delta L_{u}^{\pi}>0\right\}}\right)-\Lambda \int_{\left[0, t \wedge T_{n}\right]} \mathrm{d} R_{s}^{\pi}-M_{t \wedge T_{n}} \\
& +\mathrm{e}^{-q\left(t \wedge T_{n}\right)}\left(\frac{\Lambda \psi^{\prime}(0+)}{q}+Z^{(q)}\left(c_{2}^{\Lambda}\right)\left(\zeta\left(c_{2}^{\Lambda}\right)\right)\right)
\end{aligned}
$$

where the last inequality follows from Remark 9. In addition, by the compensation formula (see, e.g., Corollary 4.6 of Kyprianou (2014)), $\left(M_{t \wedge T_{n}}: t \geq 0\right)$ is a zero-mean $\mathbb{P}_{x}$-martingale. Now, taking expected value in Equation (29) and letting $\left(t \wedge T_{n}\right) \nearrow \infty \mathbb{P}_{x}$-a.s., the monotone convergence theorem, applied separately for $\mathbb{E}_{x}\left[\int_{\left[0, t \wedge T_{n}\right]} \mathrm{e}^{-q s} \mathrm{~d}\left(L_{s}^{\pi}-\delta \sum_{0 \leq u \leq s} 1_{\left\{\Delta L_{u}^{\pi}>0\right\}}\right)\right]$ and $\mathbb{E}_{x}\left(\Lambda \int_{\left[0, t \wedge T_{n}\right]} \mathrm{e}^{-q s} \mathrm{~d} R_{s}^{\pi}\right)$, gives

$$
v_{\delta, \Lambda}(x) \geq \mathbb{E}_{x}\left(\int_{[0, \infty)} \mathrm{e}^{-q s} \mathrm{~d}\left(L_{s}^{\pi}-\delta \sum_{0 \leq u \leq s} 1_{\left\{\Delta L_{u}^{\pi}>0\right\}}\right)-\Lambda \int_{[0, \infty)} \mathrm{e}^{-q s} \mathrm{~d} R_{s}^{\pi}\right)=v_{\delta, \Lambda}^{\pi}(x) .
$$

This completes the proof.

\section{Optimal Dividends with Capital Injection Constraint}

In this section, we are interested in maximizing the expected NPV of the dividend strategy subject to a constraint in the expected present value of the injected capital. Specifically, we aim to solve

$$
V_{\delta}(x, K):=\sup _{\pi \in \Theta_{\delta}} \mathbb{E}_{x}\left[\int_{0}^{\infty} \mathrm{e}^{-q t} \mathrm{~d}\left(L_{t}^{\pi}-\delta \sum_{0 \leq s<t} \mathbf{1}_{\left\{\Delta L_{s}^{\pi}>0\right\}}\right)\right] \quad \text { s.t. } \mathbb{E}_{x}\left[\int_{0}^{\infty} \mathrm{e}^{-q t} \mathrm{~d} R_{t}^{\pi}\right] \leq K,
$$

for any $x \geq 0$ and $K \geq 0$. Strategies $\pi$ that do not satisfy the capital injection constraint are called infeasible. Recall that the insurance company has to inject capital to ensure the non-negativity of the risk process. Therefore, small values of $K$ require very low dividend payments to keep the risk process non-negative, or would even make the problem infeasible. In the latter case, we define the value function as $-\infty$. 
To solve this problem, we use the solution of the optimal dividend problem with capital injection found in the section above. Thus, for $\Lambda \geq 0$, we define the function

$$
v_{\delta, \Lambda}^{\pi}(x, K):=v_{\delta, \Lambda}^{\pi}(x)+\Lambda K
$$

with $v_{\delta, \Lambda}^{\pi}$ as in Equation (2). It is easy to check that $V_{\delta}(x, K)=\sup _{\pi \in \Theta_{\delta}} \inf _{\Lambda \geq 0} v_{\delta, \Lambda}^{\pi}(x, K)$ since for infeasible strategies $\inf _{\Lambda \geq 0} v_{\delta, \Lambda}^{\pi}(x, K)=-\infty$. By interchanging the sup with the inf we obtain an upper bound for $V_{\delta}(x, K)$, the so-called weak duality. Hence, the dual problem of Equation (30) is defined as

$$
V_{\delta}^{D}(x, K):=\inf _{\Lambda \geq 0} \sup _{\pi \in \Theta_{\delta}} v_{\delta, \Lambda}^{\pi}(x, K)=\inf _{\Lambda \geq 0}\left\{\Lambda K+\sup _{\pi \in \Theta_{\delta}} v_{\delta, \Lambda}^{\pi}(x)\right\}=\inf _{\Lambda \geq 1}\left\{\Lambda K+V_{\delta, \Lambda}(x)\right\},
$$

with $V_{\delta, \Lambda}$ given in Equation (3). The last equality in Equation (31) is true, since $V_{\delta, \Lambda}(x)$ is infinite for any $\Lambda<1$; see Remark 2. The main goal is to prove that $V_{\delta}^{D}(x, K) \leq V_{\delta}(x, K)$.

\subsection{No Transaction Cost}

In this subsection, we consider the problem in Equation (30) without transaction cost, i.e., $\delta=0$. For this case, we denote $V(x, K):=V_{0}(x, K)$ and $V^{D}(x, K):=V_{0}^{D}(x, K)$. From Section 3.2, recall that for each $\Lambda \geq 1$, the optimal strategy is a barrier strategy, which is determined by $a_{\Lambda}$ defined in Equation (16), and its NPV satisfies $V_{\Lambda}=v_{\Lambda}^{a_{\Lambda}}$, where $v_{\Lambda}^{a_{\Lambda}}$ is as in Equation (13). Given a barrier strategy at $a>0$ and $x \in[0, a]$, the expected NPV of the injected capital is given by the function

$$
\Psi_{x}(a):=\mathbb{E}_{x}\left[\int_{0}^{\infty} \mathrm{e}^{-q t} \mathrm{~d} R_{t}^{a, 0}\right]=\frac{Z^{(q)}(a)}{q W^{(q)}(a)} Z^{(q)}(x)-k^{(q)}(x),
$$

with $k^{(q)}$ as in Equation (10). Clearly, if $x>a$, then $\Psi_{x}(a)=\Psi_{a}(a)$. We also define

$$
\underline{K}_{x}:=\lim _{a \rightarrow \infty} \Psi_{x}(a) .
$$

Using Equation (12) and the properties of scale functions (see Remark 3 (3)),

$$
\underline{K}_{x}=-k^{(q)}(x)+\frac{Z^{(q)}(x)}{\Phi(q)} .
$$

Note that $\underline{K}_{x}$ is the expected present value of the injected capital for the pay-nothing strategy $\pi_{P N}:=\left\{0, R^{0}\right\}$. Therefore, letting $a \rightarrow \infty$ in Equation (13), it can be verified

$$
v_{\Lambda}^{\pi_{P N}}(x, K)=\Lambda\left(K-\underline{K}_{x}\right) .
$$

Hence, if $K \geq \underline{K}_{x}$, then for any $x \geq 0$,

$$
V(x, K)=\sup _{\pi \in \Theta} \inf _{\Lambda \geq 0} v_{\Lambda}^{\pi}(x, K) \geq \inf _{\Lambda \geq 0} v_{\Lambda}^{\pi_{P N}}(x, K)=0 .
$$

Conversely, if $K<\underline{K}_{x}$, the problem in Equation (30) is infeasible, which is verified below.

Lemma 4. If $K<\underline{K}_{x}$, then $V(x, K)=-\infty$.

Proof. First, by Remark 7 and Equation (11), it is easy to verify that

$$
\lim _{\Lambda \rightarrow \infty} \mathbb{E}_{x}\left[\int_{0}^{\infty} \mathrm{e}^{-q t} \mathrm{~d} L_{t}^{a_{\Lambda}, 0}\right]=0, \quad \text { for } x \geq 0
$$


Then,

$$
\begin{aligned}
V^{D}(x, K) & =\inf _{\Lambda \geq 1}\left\{\Lambda K+V_{\Lambda}(x)\right\} \\
& =\inf _{\Lambda \geq 1}\left\{\mathbb{E}_{x}\left[\int_{0}^{\infty} \mathrm{e}^{-q t} \mathrm{~d} L_{t}^{a_{\Lambda}, 0}\right]+\Lambda\left(K-\Psi_{x}\left(a_{\Lambda}\right)\right)\right\} \\
& \leq \lim _{\Lambda \rightarrow \infty}\left\{\mathbb{E}_{x}\left[\int_{0}^{\infty} \mathrm{e}^{-q t} \mathrm{~d} L_{t}^{a_{\Lambda}, 0}\right]+\Lambda\left(K-\Psi_{x}\left(a_{\Lambda}\right)\right)\right\}=-\infty
\end{aligned}
$$

Now, since $V(x, K) \leq V^{D}(x, K)$ for any $x \geq 0, K \geq 0$, we have the result.

The next lemma allows us to prove that, when $K=\underline{K}_{x}$, Equation (34) holds with equality, and it is used to prove the main result of this subsection.

Lemma 5. Let $x \geq 0$ be fixed. The function $\Psi_{x}$ is strictly decreasing on $(0, \infty)$.

Proof. First, consider the case when $x<a$. Then, by Remark 4 (i), we have that $\frac{q W^{(q)}(a)}{Z^{(q)}(a)}$ is strictly increasing and the lemma is obtained. Now, when $x \geq a>0$, a simple calculation shows that

$$
\frac{\mathrm{d} \Psi_{a}(a)}{\mathrm{d} a}=-\frac{Z^{(q)}(a)\left(W^{(q) \prime}(a) Z^{(q)}(a)-q\left[W^{(q)}(a)\right]^{2}\right)}{q\left[W^{(q)}(a)\right]^{2}}=-\frac{Z^{(q)}(a) W^{(q) \prime}(a)}{q\left[W^{(q)}(a)\right]^{2}} H(a),
$$

which is strictly negative, by Remarks 3 and 5 . From here, we conclude the assertion of the lemma.

Lemma 6. If $K=\underline{K}_{x}$, then $V(x, K)=0$ and the optimal strategy is the pay-nothing strategy $\pi_{P N}$.

Proof. By Equation (34), we know that $V(x, K) \geq 0$. On the other hand, from Lemma 5 and Equation (33), we have that $\Lambda\left(K-\Psi_{x}\left(a_{\Lambda}\right)\right) \leq 0$ for all $\Lambda \geq 0$. Then, using Equations (33) and (35)

$$
\begin{aligned}
V^{D}(x, K)=\inf _{\Lambda \geq 1}\left\{\Lambda K+V_{\Lambda}(x)\right\} & =\inf _{\Lambda \geq 1}\left\{\mathbb{E}_{x}\left[\int_{0}^{\infty} \mathrm{e}^{-q t} \mathrm{~d} L_{t}^{a_{\Lambda}, 0}\right]+\Lambda\left(K-\Psi_{x}\left(a_{\Lambda}\right)\right)\right\} \\
& \leq \lim _{\Lambda \rightarrow \infty} \mathbb{E}_{x}\left[\int_{0}^{\infty} \mathrm{e}^{-q t} \mathrm{~d} L_{t}^{a_{\Lambda}, 0}\right]=0 .
\end{aligned}
$$

Now, we define

$$
\bar{K}:=\lim _{a \rightarrow 0} \Psi_{a}(a) .
$$

Using Equation (12), we have that $\bar{K}=\infty$ when the risk process has unbounded variation. Otherwise, by Remark 3 (2),

$$
\bar{K}=\frac{c-\psi^{\prime}(0+)}{q}
$$

and $\bar{K}$ corresponds to the expected NPV of the injected capital for the strategy $\pi_{0,0}$ (see Equation (4.5) in Avram et al. (2007)).

Lemma 7. Assume that the risk process $X$ has bounded variation. If $K \geq \bar{K}$, then $V(x, K)=K+V_{1}(x)$, with $V_{1}(x)=x+\frac{\psi^{\prime}(0+)}{q}$. 
Proof. If the Lévy measure is finite, by Equation (16), we have that $a_{1}=0$. The same is true for the infinite Lévy measure case since $H^{-1}(1)=0$ by Remark 5 . Using Equation (13) and Remark 6, we obtain

$$
V_{1}(x)=v_{1}^{0}(x)=x+\frac{c}{q}-\bar{K}=x+\frac{\psi^{\prime}(0+)}{q}, \quad \text { for } x \geq 0 .
$$

Now, by Equations (31), (36) and (37) and the weak duality, we get

$$
V(x, K) \leq V^{D}(x, K) \leq K+v_{1}^{0}(x)=K+V_{1}(x) .
$$

Since $K \geq \bar{K}, \pi_{0,0}$ is a feasible strategy. Then, using Equation (11), it yields,

$$
V(x, K) \geq \inf _{\Lambda \geq 1}\left\{v_{\Lambda}^{0}(x)+\Lambda K\right\}=x+K-\frac{c-\psi^{\prime}(0+)}{q}+\frac{c}{q}=K+V_{1}(x) .
$$

Therefore, $V(x, K)=K+V_{1}(x)$.

We are now ready for the main result of this subsection.

Theorem 2. Assume $\delta=0$ and let $V$ and $V^{D}$ as in Equation (30) and Equation (31), respectively, then $V=V^{D}$. Furthermore, if $x$ and $K$ are such that $K \in\left(\underline{K}_{x}, \bar{K}\right)$, then

$$
V(x, K)=\Lambda^{*} K+V_{\Lambda^{*}}(x)=\mathbb{E}_{x}\left[\int_{0}^{\infty} \mathrm{e}^{-q t} \mathrm{~d} L_{t}^{a^{*}, 0}\right],
$$

where $a^{*}=\Psi_{x}^{-1}(K)$, and $\Lambda^{*}=\frac{1}{H\left(a^{*}\right)}$.

Proof. Lemmas 4, 6 and 7 show imply that Equation (38) holds when $x$ and $K$ are such that $K \in$ $\left[0, \underline{K}_{x}\right] \cup[\bar{K}, \infty)$. Assume now that $K \in\left(\underline{K}_{x}, \bar{K}\right)$, then by Lemma 5 the function $\Psi_{x}$ is injective, so there exists a unique $a^{*}>0$ such that $\Psi_{x}\left(a^{*}\right)=K$. Note that from Equation (16), we have that there exists a unique $\Lambda^{*}$ such that $a_{\Lambda^{*}}=a^{*}$. Then,

$$
\begin{aligned}
V^{D}(x, K) & \leq \Lambda^{*} K+V_{\Lambda^{*}}(x) \\
& =\Lambda^{*} K+\mathbb{E}_{x}\left[\int_{0}^{\infty} \mathrm{e}^{-q t} \mathrm{~d} L_{t}^{a^{*}, 0}\right]-\Lambda^{*} \Psi_{x}\left(a^{*}\right) \\
& =\mathbb{E}_{x}\left[\int_{0}^{\infty} \mathrm{e}^{-q t} \mathrm{~d} L_{t}^{a^{*}, 0}\right] .
\end{aligned}
$$

Meanwhile, since the strategy $\pi_{a^{*}, 0}$ is feasible, we see

$$
\begin{aligned}
V(x, K) \geq \inf _{\Lambda \geq 1}\left\{v_{\Lambda}^{\pi_{a^{*}, 0}}(x)+\Lambda K\right\} & =\inf _{\Lambda \geq 1}\left\{\mathbb{E}_{x}\left[\int_{0}^{\infty} \mathrm{e}^{-q t} \mathrm{~d} L_{t}^{a^{*}, 0}\right]+\Lambda\left(K-\Psi_{x}\left(a^{*}\right)\right)\right\} \\
& =\mathbb{E}_{x}\left[\int_{0}^{\infty} \mathrm{e}^{-q t} \mathrm{~d} L_{t}^{a^{*}, 0}\right] .
\end{aligned}
$$

This implies that $V^{D}(x, K) \leq V(x, K)$. Finally, the weak duality gives Equation (46).

\subsection{With Transaction Cost}

Now, we consider the problem given in Equation (30) with transaction $\operatorname{cost} \delta>0$. From the previous section, we know that optimal strategies are $\left(c_{1}^{\Lambda}, c_{2}^{\Lambda}\right)$-reflected strategies with $\left(c_{1}^{\Lambda}, c_{2}^{\Lambda}\right)$ given in Proposition 2.

Proposition 4. The curve $\Lambda \mapsto\left(c_{1}^{\Lambda}, c_{2}^{\Lambda}\right)$ is continuous and unbounded, for $\Lambda \in[1, \infty)$. 
Proof. From Remark 7 and the fact that $a_{\Lambda}<c_{2}^{\Lambda}$ (by Proposition 2), we know that $c_{2}^{\Lambda} \rightarrow \infty$ as $\Lambda \rightarrow \infty$, so the curve is unbounded. To show the continuity of the curve, we consider two cases and use the implicit function theorem. To this end, suppose first $c_{1}^{\Lambda}=0$. Defining $f\left(\Lambda, c_{2}\right):=G_{\Lambda}\left(0, c_{2}\right)-\zeta_{\Lambda}\left(c_{2}\right)$, we have $f\left(\Lambda, c_{2}^{\Lambda}\right)=0$. Then,

$$
\frac{\partial f}{\partial c_{2}}\left(\Lambda, c_{2}^{\Lambda}\right)=\frac{\partial G_{\Lambda}}{\partial c_{2}}\left(0, c_{2}^{\Lambda}\right)-\zeta_{\Lambda}^{\prime}\left(c_{2}^{\Lambda}\right)=-\zeta_{\Lambda}^{\prime}\left(c_{2}^{\Lambda}\right)>0,
$$

since $c_{2}^{\Lambda}>a_{\Lambda}$. From here, we see that the conditions of the implicit function theorem are satisfied. Now, if $c_{1}^{\Lambda}>0$, define the function $f\left(\Lambda, c_{1}, c_{2}\right)=\left(f_{1}\left(\Lambda, c_{1}, c_{2}\right), f_{2}\left(\Lambda, c_{1}, c_{2}\right)\right)$ by

$$
\begin{aligned}
& f_{1}\left(\Lambda, c_{1}, c_{2}\right):=G_{\Lambda}\left(c_{1}, c_{2}\right)-\zeta_{\Lambda}\left(c_{1}\right), \\
& f_{2}\left(\Lambda, c_{1}, c_{2}\right):=G_{\Lambda}\left(c_{1}, c_{2}\right)-\zeta_{\Lambda}\left(c_{2}\right) .
\end{aligned}
$$

Then, $f\left(\Lambda, c_{1}^{\Lambda}, c_{2}^{\Lambda}\right)=(0,0)$. Again, simple calculations show that the Jacobian determinant of this system of equations is $\zeta_{\Lambda}^{\prime}\left(c_{2}^{\Lambda}\right) \zeta_{\Lambda}^{\prime}\left(c_{1}^{\Lambda}\right)<0$, since $c_{1}^{\Lambda}<a_{\Lambda}<c_{2}^{\Lambda}$. Therefore, the curve $\Lambda \mapsto\left(c_{1}^{\Lambda}, c_{2}^{\Lambda}\right)$ is continuous, for $\Lambda \in[1, \infty)$.

Next, we analyze the level curves of the constraint. Let $\bar{\Psi}_{x}\left(c_{1}, c_{2}\right)$ be the expected present value of the injected capital under a $\left(c_{1}, c_{2}\right)$-reflected policy. Then, the calculations given in the proof of Lemma 1 show that

$$
\begin{aligned}
\bar{\Psi}_{x}\left(c_{1}, c_{2}\right):= & \mathbb{E}_{x}\left[\int_{0}^{\infty} \mathrm{e}^{-q t} \mathrm{~d} R_{t}^{\left(c_{1}, c_{2}\right), 0}\right] \\
& = \begin{cases}Z^{(q)}(x) \frac{\bar{Z}^{(q)}\left(c_{2}\right)-\bar{Z}^{(q)}\left(c_{1}\right)}{Z^{(q)}\left(c_{2}\right)-Z^{(q)}\left(c_{1}\right)}-k^{(q)}(x), & \text { if } 0 \leq x \leq c_{2}, \\
\frac{\bar{Z}^{(q)}\left(c_{2}\right) Z^{(q)}\left(c_{1}\right)-\bar{Z}^{(q)}\left(c_{1}\right) Z^{(q)}\left(c_{2}\right)}{Z^{(q)}\left(c_{2}\right)-Z^{(q)}\left(c_{1}\right)}-\frac{\psi^{\prime}(0+)}{q}, & \text { if } x>c_{2} .\end{cases}
\end{aligned}
$$

Remark 11. Note that $\lim _{c_{1} \rightarrow c_{2}} \bar{\Psi}_{x}\left(c_{1}, c_{2}\right)=\Psi_{x}\left(c_{2}\right)$, where $\Psi_{x}$ is as in Equation (32).

The next lemmas describe some properties of $\bar{\Psi}_{x}\left(c_{1}, c_{2}\right)$.

Lemma 8. Let $x \geq 0$ be fixed.

1. If $c_{1} \geq 0$ is fixed, then the function $\bar{\Psi}_{x}\left(c_{1}, c_{2}\right)$, given in Equation (39), is strictly decreasing for all $c_{2}>c_{1}$, and

$$
\lim _{c_{2} \rightarrow \infty} \bar{\Psi}_{x}\left(c_{1}, c_{2}\right)=\underline{K}_{x}
$$

where $\underline{K}_{x}$ is defined in Equation (33).

2. If $c_{2}>0$ is fixed, $\bar{\Psi}_{x}\left(c_{1}, c_{2}\right)$ is strictly decreasing for all $c_{1} \in\left[0, c_{2}\right)$.

Proof. Let $c_{1} \geq 0$ be fixed. First, assume that $c_{2} \geq x$. To show that $\bar{\Psi}_{x}\left(c_{1}, c_{2}\right)$ is strictly decreasing, it is sufficient to verify that

$$
\frac{\bar{Z}^{(q)}\left(c_{2}\right)-\bar{Z}^{(q)}\left(c_{1}\right)}{Z^{(q)}\left(c_{2}\right)-Z^{(q)}\left(c_{1}\right)}
$$

is strictly decreasing, which is true if

$$
\frac{\partial}{\partial c_{2}}\left[\frac{\bar{Z}^{(q)}\left(c_{2}\right)-\bar{Z}^{(q)}\left(c_{1}\right)}{Z^{(q)}\left(c_{2}\right)-Z^{(q)}\left(c_{1}\right)}\right]=\frac{Z^{(q)}\left(c_{2}\right)}{Z^{(q)}\left(c_{2}\right)-Z^{(q)}\left(c_{1}\right)}-\frac{q W^{(q)}\left(c_{2}\right)\left(\bar{Z}^{(q)}\left(c_{2}\right)-\bar{Z}^{(q)}\left(c_{1}\right)\right)}{\left[Z^{(q)}\left(c_{2}\right)-Z^{(q)}\left(c_{1}\right)\right]^{2}}<0 .
$$


Since $Z^{(q)}$ is a strictly log-convex function on $[0, \infty)$ by Remark 4 (i),

$$
\frac{q W^{(q)}(\eta)}{Z^{(q)}(\eta)}<\frac{q W^{(q)}(\varsigma)}{Z^{(q)}(\varsigma)}, \text { for } \eta \text { and } \varsigma \text { such that } \eta<\varsigma
$$

Taking $\varsigma=c_{2}$ in the inequality above and integrating between $c_{1}$ and $c_{2}$, it follows that

$$
Z^{(q)}\left(c_{2}\right)<\frac{q W^{(q)}\left(c_{2}\right)\left[\bar{Z}^{(q)}\left(c_{2}\right)-\bar{Z}^{(q)}\left(c_{1}\right)\right]}{Z^{(q)}\left(c_{2}\right)-Z^{(q)}\left(c_{1}\right)} .
$$

Then, Equation (43) yields Equation (42) and hence Equation (41) is strictly decreasing. For the case $x>c_{2}$, it can be verified that

$$
\begin{aligned}
\frac{\partial}{\partial c_{2}}\left[Z^{(q)}\left(c_{2}\right) \frac{\bar{Z}^{(q)}\left(c_{2}\right)-\bar{Z}^{(q)}\left(c_{1}\right)}{Z^{(q)}\left(c_{2}\right)-Z^{(q)}\left(c_{1}\right)}-\bar{Z}^{(q)}\left(c_{2}\right)\right] \\
=\frac{Z^{(q)}\left(c_{1}\right)}{Z^{(q)}\left(c_{2}\right)-Z^{(q)}\left(c_{1}\right)}\left[Z^{(q)}\left(c_{2}\right)-\frac{q W^{(q)}\left(c_{2}\right)\left[\bar{Z}^{(q)}\left(c_{2}\right)-\bar{Z}^{(q)}\left(c_{1}\right)\right]}{Z^{(q)}\left(c_{2}\right)-Z^{(q)}\left(c_{1}\right)}\right] .
\end{aligned}
$$

Then, using Equations (43) and (44), we obtain that $\bar{\Psi}_{x}\left(c_{1}, c_{2}\right)$ is strictly decreasing for all $c_{2} \in$ $\left(c_{1}, x\right)$. Similarly, we obtain Point 2 of the lemma. Now, by L'Hôpital's rule together with Exercise 8.5 (i) in Kyprianou (2014), it is not difficult to see that Equation (40) holds for any $c_{1} \geq 0$.

Note that Equation (34) still holds if $K \geq \underline{K}_{x}$. On the other hand, using that $c_{2}^{\Lambda} \rightarrow \infty$ as $\Lambda \rightarrow \infty$ together with Equation (18) we have that

$$
\lim _{\Lambda \rightarrow \infty} \mathbb{E}_{x}\left[\int_{0}^{\infty} \mathrm{e}^{-q t} \mathrm{~d} L_{t}^{\left(c_{1}^{\Lambda}, c_{2}^{\Lambda}\right), 0}\right]=\lim _{\Lambda \rightarrow \infty}\left(c_{2}^{\Lambda}-c_{1}^{\Lambda}-\delta\right) \frac{Z^{(q)}(x)}{Z^{(q)}\left(c_{2}^{\Lambda}\right)-Z^{(q)}\left(c_{1}^{\Lambda}\right)}=0,
$$

by Remark 3 (3).

Remark 12. Using the same arguments as in Lemma 7 , we have that $c_{1}^{1}=a_{1}=0<c_{2}^{1}$ for bounded and unbounded variation processes. Similarly, if $x$ and $K$ are such that $K \geq \bar{\Psi}_{x}\left(0, c_{2}^{1}\right)=: \bar{K}_{x}$, then $V_{\delta}(x, K)=$ $V_{\delta, 1}(x)+K$. Note also that $\bar{K}_{x}<\bar{K}$.

Lemma 9. Let $x \geq 0$ be fixed. Then, for each $K \in\left(\underline{K}_{x}, \bar{K}\right)$, there exist $\underline{c} \leq \bar{c}$ such that the level curve $L_{K}\left(\bar{\Psi}_{x}\right):=\left\{\left(c_{1}, c_{2}\right): \bar{\Psi}_{x}\left(c_{1}, c_{2}\right)=K\right\}$ is continuous, contained in the set $[0, \underline{c}] \times[\underline{c}, \bar{c}]$ and contains the points $(0, \bar{c})$ and $(\underline{c}, \bar{c})$.

Proof. The continuity of the level curve is obtained as an immediate consequence of the continuity of $\bar{\Psi}_{x}$. Observe that, by Lemma 5, we know the existence of $\underline{c}>0$ such that $\Psi_{x}(\underline{c})=K$. Meanwhile, from Lemma 8, we have that there exists $\bar{c} \in[\underline{c}, \infty)$ such that $\bar{\Psi}_{x}(0, \bar{c})=K$. Now, the fact that the level curve $L_{K}\left(\bar{\Psi}_{x}\right)$ is contained in $[0, \underline{c}] \times[\underline{c}, \bar{c}]$ is a consequence of Remark 11 and Lemma 8.

Remark 13. Lemmas 4 and 9 yield that the parametric curve $\Lambda \mapsto\left(c_{1}^{\Lambda}, c_{2}^{\Lambda}\right)$ and the level curve $L_{K}\left(\bar{\Psi}_{x}\right)$ must intersect, i.e., there exists $\Lambda^{*}$ such that $\bar{\Psi}_{x}\left(c_{1}^{\Lambda^{*}}, c_{2}^{\Lambda^{*}}\right)=K$, for $K \in\left(\underline{K}_{x}, \bar{K}_{x}\right]$.

By similar arguments as in the proof of Theorem 2, by Remarks 12 and 13, and using Equation (45), we get the following result, whose proof is omitted.

Theorem 3. Assume $\delta>0$ and let $V_{\delta}$ and $V_{\delta}^{D}$ as in Equations (30) and (31), respectively, then $V_{\delta}=V_{\delta}^{D}$. Furthermore, if $x, K$ are such that

1. $K<\underline{K}_{x}$, then $V_{\delta}(x, K)=-\infty$; 
2. $K=\underline{K}_{x}$, then $V_{\delta}(x, K)=0$;

3. $K \geq \bar{K}_{x}$, then $V_{\delta}(x, K)=V_{\delta, 1}(x)+K$; and

4. $K \in\left(\underline{K}_{x}, \bar{K}_{x}\right)$, then there exists $\Lambda^{*} \geq 1$ such that

$$
V_{\delta}(x, K)=\Lambda^{*} K+V_{\delta, \Lambda^{*}}(x)=\mathbb{E}_{x}\left[\int_{0}^{\infty} \mathrm{e}^{-q t} \mathrm{~d}\left(L_{t}^{\left(c_{1}^{\Lambda^{*}}, c_{2}^{\Lambda^{*}}\right), 0}-\delta \sum_{0 \leq s<t} \mathbf{1}\left\{\Delta L_{s}^{\left(c_{1}^{*}, c_{2}^{\Lambda^{*}}\right), 0}>0\right\}\right)\right] .
$$

\section{Numerical Examples}

In this section, we confirm the obtained results by a sequence of numerical examples. Here, we assume that $X$ is of the form

$$
X_{t}-X_{0}=t+0.5 B_{t}-\sum_{n=1}^{N_{t}} Z_{n}, \quad 0 \leq t<\infty,
$$

where $B=\left\{B_{t}: t \geq 0\right\}, N=\left\{N_{t}: t \geq 0\right\}$, and $Z=\left\{Z_{n}\right\}_{n \geq 1}$ are a standard Brownian motion, a Poisson process with arrival rate $\lambda=0.4$, and an i.i.d. sequence of random variables with distribution Gamma $(1,2)$, respectively, which are assumed mutually independent. Since there is no closed form for the scale function $W^{(q)}$ associated with $X$, we use a numerical algorithm presented in Surya (2008) in order to approximate the inverse Laplace transform of Equation (4). Similarly, we approximate the derivatives of the scale functions and use the trapezoidal rule to calculate its integrals.

We first consider the case without transaction cost presented in Section 3.2. In Figure 1 (left), we plot the function $x \mapsto V_{\Lambda}(x)+\Lambda K$ for various values of $\Lambda$ and a fixed value of $K$. For $x \geq x_{0}$, where $x_{0}$ is such that $\underline{K}_{x_{0}}=K$, its minimum over the considered values of $\Lambda$ provides (an approximation of) $V(x, K)$, indicated by the solid red line in the plot. Since the process has unbounded variation, then $\bar{K}=\infty$. In Figure 1 (right), we plot, for $x>x_{0}$, the Lagrange multiplier $\Lambda^{*}$ given in Theorem 2 . We observe that $\Lambda^{*}$ goes to infinity as $x \downarrow x_{0}$ and remains always above 1 .
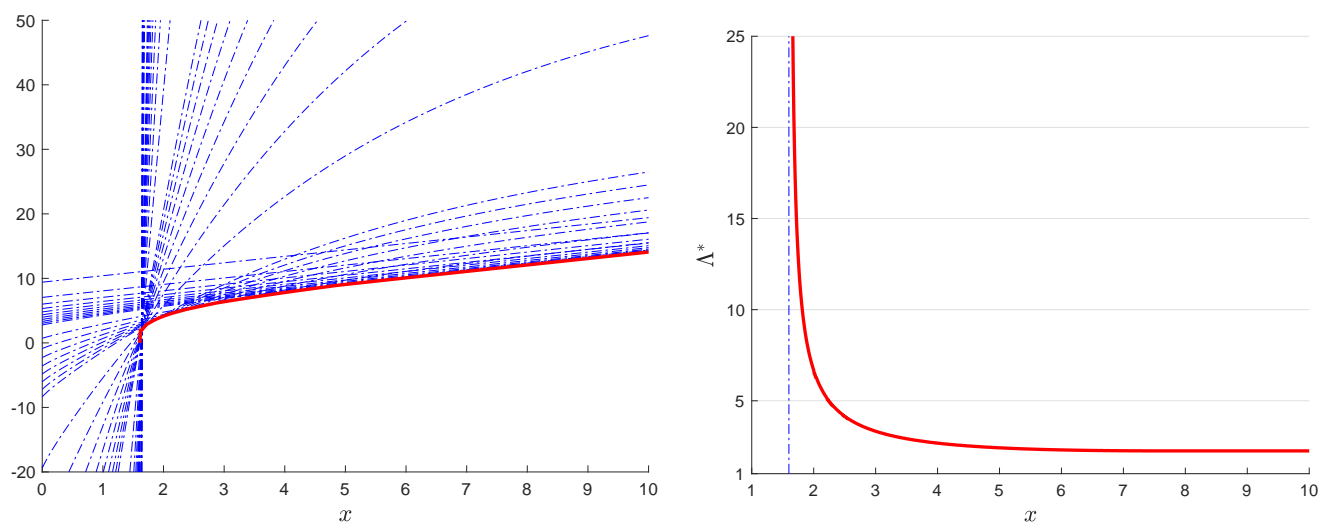

Figure 1. (Left) Plots of $x \mapsto V_{\Lambda}(x)+\Lambda K$ for $\Lambda=1,1.1, \ldots, 2,3, \ldots, 10$, $20, \ldots, 100,200, \ldots, 1000,2000, \ldots, 10,000,20,000$ (dotted) for the case $K=2.7$. The minimum of $V_{\Lambda}(x)+\Lambda K$ over $\Lambda$ is shown in solid bold-face red line. (Right) Plot of the Lagrange multiplier $\Lambda^{*}$ for $x>x_{0}$, where $x_{0}$ is such that $\underline{K}_{x_{0}}=K$.

In Figure 2, we show the values of $V(x, K)$ and Lagrange multiplier $\Lambda^{*}$ as functions of $(x, K)$. It is confirmed that $V(x, K)$ increases as $x$ and $K$ increase, while $\Lambda^{*}$ increases as $x$ and $K$ decrease. 

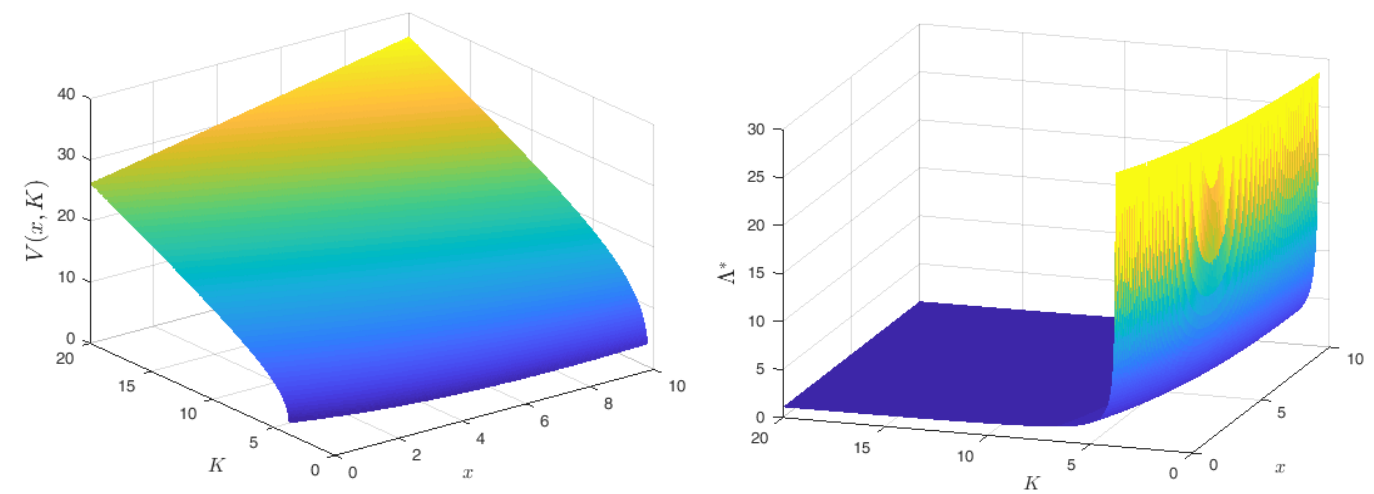

Figure 2. Plots of $V(x, K)$ (left); and the Lagrange multiplier $\Lambda^{*}($ right) as functions of $x$ and $K$.

We now move to the case with transaction cost. First, we illustrate the results shown in Section 4. In Figure 3 (left), we plot the function $x \mapsto \zeta_{\Lambda}(x)$ for the values of $\Lambda=1, \ldots, 9$. We also plot its maximum value attained at $a_{\Lambda}$ and the value attained at the corresponding optimal values $\left(c_{1}^{\Lambda}, c_{2}^{\Lambda}\right)$ with transaction $\operatorname{cost} \delta=0.05$. Note that, when $\Lambda=1, a_{\Lambda}=c_{1}^{\Lambda}=0$ and for the other values of $\Lambda$, $\zeta_{\Lambda}\left(c_{1}^{\Lambda}\right)=\zeta_{\Lambda}\left(c_{2}^{\Lambda}\right)<\zeta_{\Lambda}\left(a_{\Lambda}\right)$. In Figure 3 (right), we plot the optimal thresholds $a_{\Lambda}, c_{1}^{\Lambda}$ and,$c_{2}^{\Lambda}$ as function of $\Lambda$.
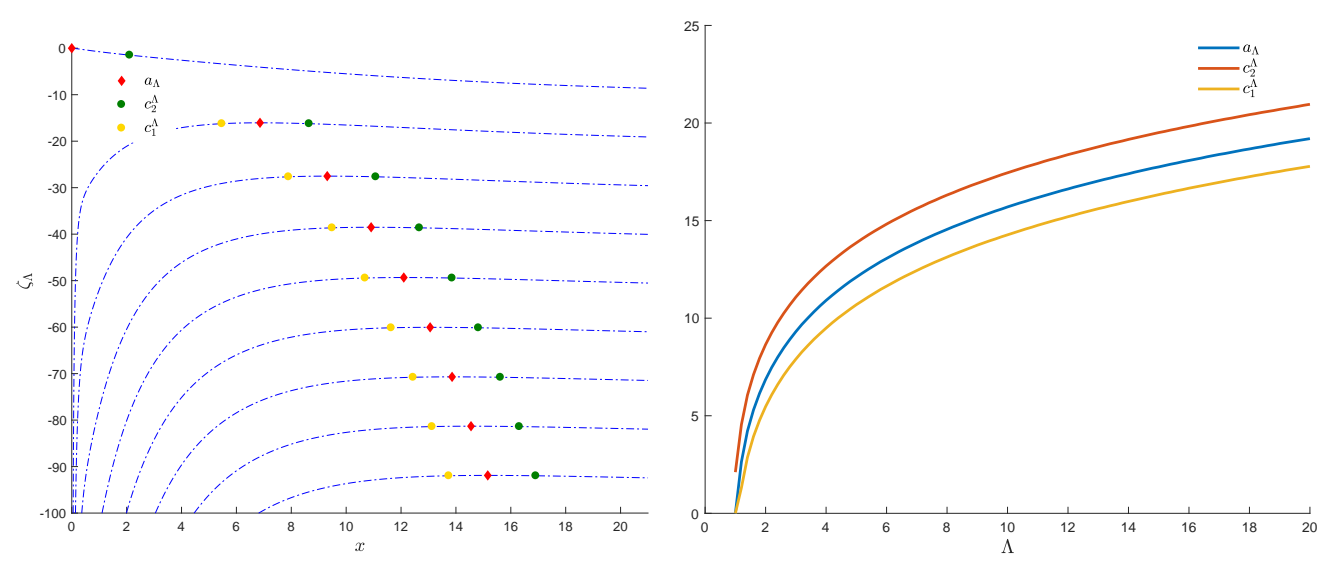

Figure 3. (Left) Plots of $x \mapsto \zeta_{\Lambda}(x)$ for $\Lambda=1, \ldots, 9$ and the corresponding values of $a_{\Lambda}, c_{1}^{\Lambda}$ and,$c_{2}^{\Lambda}$ for $\delta=0.05$. (Right) Plots of the functions $\Lambda \mapsto a_{\Lambda}, c_{1}^{\Lambda}$ and,$c_{2}^{\Lambda}$.

In Figure 4, we illustrate the findings of Section 5.2. This figure is analogous to Figure 1 but with transaction cost $\delta$ as above. It can be seen that the change in the function $V_{\delta}(x, K)$ is relatively very small, but the change in the optimal Lagrange multiplier $\Lambda^{*}$ is significant, being smaller in the case of transaction cost. A similar figure as Figure 2 in the case of transaction cost is omitted since both have the same shape. 

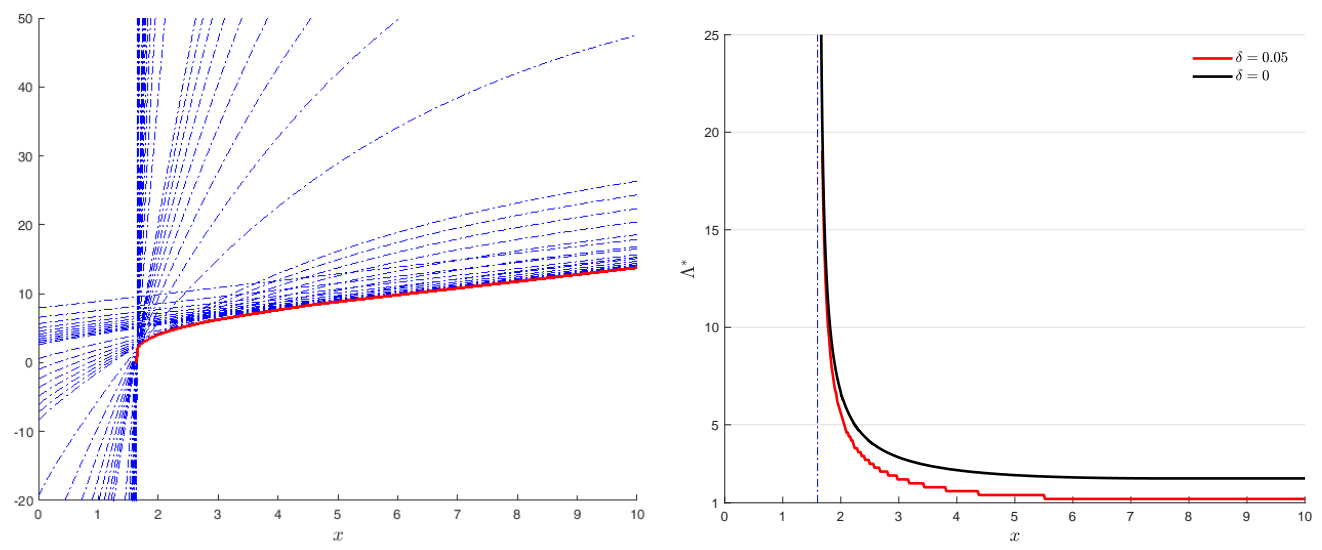

Figure 4. (Left) Plots of $x \mapsto V_{\Lambda}(x)+\Lambda K$ for $\Lambda=1,1.1, \ldots, 2,3, \ldots, 10$, $20, \ldots, 100,200, \ldots, 1000,2000, \ldots, 10,000,20,000$ (dotted) for the case $K=2.7$. The minimum of $V_{\delta, \Lambda}(x)+\Lambda K$ over $\Lambda$ is plotted in solid bold-face red line. (Right) Plots of the Lagrange multipliers $\Lambda^{*}$ for $x>x_{0}$, where $x_{0}$ is such that $\underline{K}_{x_{0}}=K$ with $\delta=0$ and $\delta=0.05$.

\section{Concluding Remarks}

In this study, we proved that the optimal strategy for the bail-out dividend problem with fixed transaction costs is given by a reflected $\left(c_{1}, c_{2}\right)$-policy. We also characterized the optimal thresholds and gave a semi-explicit form for the value function in terms of the scale functions. In addition, we used the previous results to solve the constrained dividend maximization problem with the restriction that the expected present value of the capital injected is bounded by a given constant. The solution of the constrained problem can provide the insurance company with a guideline to maximize the profits of the shareholders taking into account the risk of bail-out losses.

It is a legitimate and interesting question whether the optimal strategy and the associated value function with transaction costs (i.e., $\delta>0$ ) converge to the corresponding optimal strategy and its value function without transaction cost as $\delta \downarrow 0$. Although we conjecture that indeed this is the case, further investigation is needed.

Another interesting generalization would involve considering fixed and proportional costs for the capital injection as well. We conjecture that in this case the optimal strategy would consist in a double band strategy, that is, a band strategy similar to the dividend payment strategy given in this paper, and a band strategy for the capital injection, which consists in pushing the process to a positive level each time the surplus process tries to cross below 0 . We leave this problem as an opportunity for future research.

Author Contributions: All authors equally contributed to this paper.

Funding: This research received no external funding.

Acknowledgments: The authors would like to thank the anonymous reviewers for their comments and suggestions, which helped to improve significantly the quality of this paper. They would also like to thank Florin Avram who encouraged them to contribute to this special issue of Risks.

Conflicts of Interest: The authors declare no conflict of interest.

\section{References}

Avram, Florin, Zbigniew Palmowski, and Martin Pistorius. 2007. On the optimal dividend problem for a spectrally negative Lévy process. The Annals of Applied Probability 17: 156-80. [CrossRef]

Bayraktar, Erhan, Andreas E. Kyprianou, and Kazutoshi Yamazaki. 2014a. On optimal dividends in the dual problem. ASTIN Bulletin 43: 359-72. [CrossRef]

Bayraktar, Erhan, Andreas E. Kyprianou, and Kazutoshi Yamazaki. 2014b. Optimal dividends in the dual model under transaction costs. Insurance: Mathematics and Economics 54: 133-43. [CrossRef]

Bertoin, Jean. 1998. Lévy Processes. Cambridge: Cambridge University Press. 
Chan, Terence, Andreas E. Kyprianou, and Mladen Savov. 2011. Smoothness of scale functions for spectrally negative Lévy processes. Probability Theory and Related Fields 150: 691-708. [CrossRef]

Hernández, Camilo, Mauricio Junca, and Harold Moreno-Franco. 2018. A time of ruin constrained optimal dividend problem for spectrally one-sided Lévy processes. Insurance: Mathematics and Economics 79: 57-68. [CrossRef]

Kuznetsov, Alexey, Andreas E. Kyprianou, and Victor Rivero. 2013. The theory of scale functions for spectrally negative Lévy processes. Levy Matters II 2061: 97-186.

Kyprianou, Andreas E. 2014. Fluctuations of Léry Processes with Applications. Universitext. Berlin and Heidelberg: Springer.

Loeffen, Ronnie. 2008. On the optimality of the barrier strategy in de Finetti's problem for spectrally negative Lévy processes. The Annals of Applied Probability 18: 1669-80. [CrossRef]

Loeffen, Ronnie L. 2009. An optimal dividends problem with transaction costs for spectrally negative Lévy processes. Insurance: Mathematics and Economics 45: 41-48. [CrossRef]

Pistorius, Martijn R. 2004. On exit and ergodicity of the spectrally one-sided Lévy process reflected at its infimum. Journal of Theoretical Probability 17: 183-220. [CrossRef]

Protter, Philip. 2005. Stochastic Integration and Differential Equations, 2nd ed. Berlin: Springer.

Surya, Budhig A. 2008. Evaluating scale functions of spectrally negative Lévy processes. Journal of Applied Probability 45: 135-49. [CrossRef]

(c) 2019 by the authors. Licensee MDPI, Basel, Switzerland. This article is an open access article distributed under the terms and conditions of the Creative Commons Attribution (CC BY) license (http://creativecommons.org/licenses/by/4.0/). 